\title{
Niger: Second Review Under the Three-Year Arrangement Under the Poverty Reduction and Growth Facility and Request for Modification of Performance Criteria- Staff Report; Press Release on the Executive Board Discussion
}

In the context of the second review under the three-year arrangement under the Poverty Reduction and Growth Facility and request for a modification of performance criteria for Niger, the following documents have been released and are included in this package:

- $\quad$ The staff report for the Second Review Under the Three-Year Arrangement Under the Poverty Reduction and Growth Facility and Request for Modification of Performance Criteria, prepared by a staff team of the IMF, following discussions that ended on March 17, 2009 , with the officials of Niger on economic developments and policies. Based on information available at the time of these discussions, the staff report was completed on April 29, 2009. The views expressed in the staff report are those of the staff team and do not necessarily reflect the views of the Executive Board of the IMF.

- $\quad$ A Press Release summarizing the views of the Executive Board.

The documents listed below have been or will be separately released.

Letter of Intent sent to the IMF by the authorities of Niger*

Technical Memorandum of Understanding*

*Also included in Staff Report

The policy of publication of staff reports and other documents allows for the deletion of marketsensitive information.

\author{
Copies of this report are available to the public from \\ International Monetary Fund • Publication Services \\ $70019^{\text {th }}$ Street, N.W. $\bullet$ Washington, D.C. 20431 \\ Telephone: (202) 623-7430 • Telefax: (202) 623-7201 \\ E-mail: publications@imf.org •Internet: http://www.imf.org
}

\section{International Monetary Fund Washington, D.C.}



INTERNATIONAL MONETARY FUND

NIGER

\title{
Second Review Under the Three-Year Arrangement Under the Poverty Reduction and Growth Facility and Request for Modification of Performance Criteria
}

\author{
Prepared by the African Department \\ (In consultation with other departments) \\ Approved by T. Krueger and A. Boote
}

April 29, 2009

\begin{tabular}{|c|c|}
\hline Staff team & Mr. Sacerdoti (head), Mr. Gueye, and Mr. Salinas (all AFR). \\
\hline Discussions & Niamey, March 3-17, 2009. \\
\hline $\begin{array}{l}\text { Relations with the } \\
\text { Fund }\end{array}$ & $\begin{array}{l}\text { The PRGF arrangement (SDR } 23.03 \text { million, } 35 \text { percent of quota) was approved by } \\
\text { the Board on May } 28,2008 \text {. It expires on June } 1,2011 \text {. Niger received MDRI } \\
\text { relief in } 2006 . \text { The first review was concluded by the Executive Board on } \\
\text { December } 19,2008 \text {. }\end{array}$ \\
\hline $\begin{array}{l}\text { Key results of } \\
\text { review }\end{array}$ & $\begin{array}{l}\text { - All end-December } 2008 \text { and continuous performance criteria have been met. } \\
\text { - Staff recommends completion of the second review and the requested } \\
\text { modification of performance criteria. }\end{array}$ \\
\hline & $\begin{array}{l}\text { - GDP growth was strong in } 2008 \text {, because of a record harvest, and is likely to be } \\
\text { more moderate in } 2008 \text {, as agricultural production could decline. } \\
\text { Non-agricultural growth is likely to be sustained, despite the international } \\
\text { downturn, in light of the start of large foreign-financed projects in uranium } \\
\text { mining and petroleum. }\end{array}$ \\
\hline Political situation & $\begin{array}{l}\text { - A low level conflict between the Touareg rebels and government troops in } \\
\text { Northern Niger continues. }\end{array}$ \\
\hline
\end{tabular}




\section{CONTENTS}

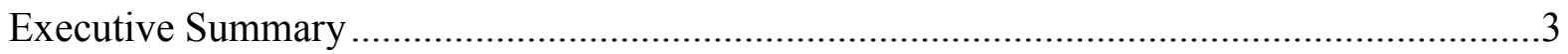

I. Recent Developments and Program Performance ….......................................................

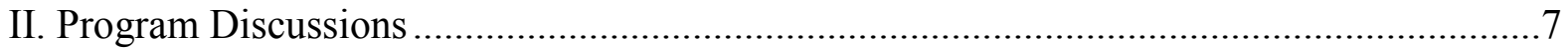

A. Macroeconomic Framework and Risk to the Outlook ......................................... 11

B. Budgetary Outlook for 2009 and Fiscal Reforms ............................................. 11

C. Financial Sector Reforms and Mining Management Issues................................12

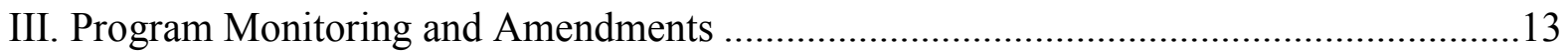

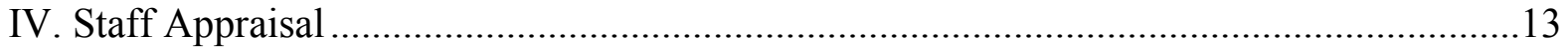

Tables

1. Selected Economic and Financial Indicators, 2006-11 f............................................14

2a. Financial Operations of the Central Government, 2006-11 (Billions of CFA francs).......15

2b. Financial Operations of the Central Government, 2006-11 (In percent of GDP).............16

2c. Financial Operations of the Central Government, 2006-11 (Quarterly

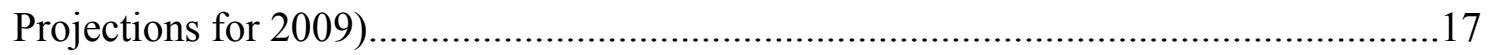

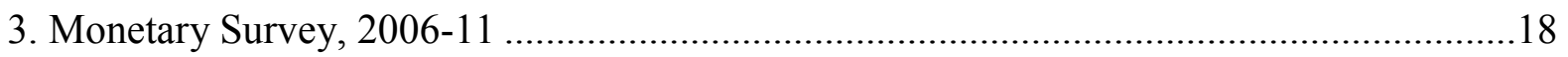

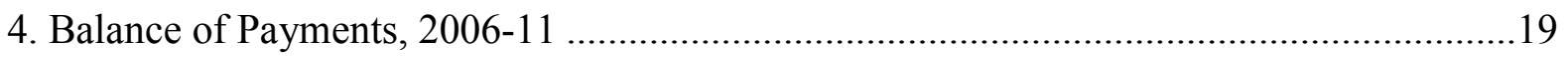

5. Budget Expenditure on Social and Rural Sectors, 2005-09..........................................20

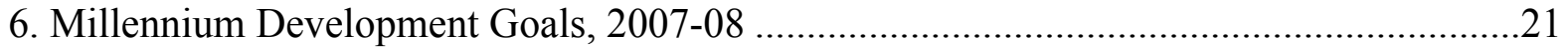

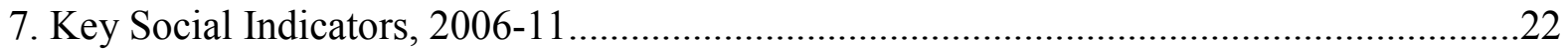

8. Proposed Scheduled Disbursements Under the PRGF Arrangement, 2008-11 _...............23

Appendix

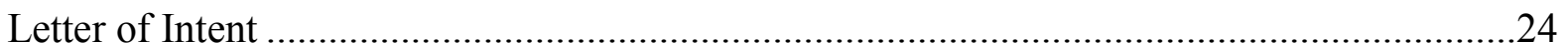

Attachment: Technical Memorandum of Understanding ............................................33 


\section{Executive Summary}

Economic developments. Niger's GDP growth is projected to decline in 2009 to 3 percent, from 9.5 percent in 2008 when agricultural production reached a record level. Growth would continue to be sustained by large mining and infrastructure projects already started; therefore the country appears relatively protected from the international downturn. Inflation is decelerating and could reach 2 percent at year-end, in light of receding food and fuel prices. Social indicators have improved markedly since 2006, reflecting the introduction of free access to health services for children under 5 and pregnant mothers. Poverty indicators also improved.

Program performance. Budgetary performance in 2008 was better than expected because of strong revenue performance and some deferment of spending to 2009. All end-2008 and continuous performance criteria were observed. The two quantitative performance criteria for end-2009 are being converted into structural benchmarks, in light of the new Fund policy in this area; their observance will be a key element of the fourth review.

Policy discussions. After the strong performance in 2008, revenue targets for 2009 have been adjusted upwards to 11.7 percent of GDP from 11.5 in the original program. Capital expenditure have been raised by the equivalent of 1 percent of GDP from the original program to reflect the deferment in 2009 of the outlays for the refinery originally envisaged in 2008. This will result in a modestly higher basic budget deficit for 2009 , by 0.5 percent of GDP. It is proposed to adjust accordingly the domestic budgetary financing criteria for end-June and end-December 2009. The authorities are continuing comprehensive public financial management reform, and reforms in the financial sector; the establishment of a new postal bank and an agricultural bank require attention to management and operational aspects, in order to avoid the pitfalls of the past. 


\section{Recent Developments and Program Performance}

1. Economic performance was very positive in 2008 with a surge in GDP growth to 9.5 percent, up from 3.3 percent in 2007 (Table 1). The strong growth rate was the result of an excellent harvest, that brought about a 25 percent increase in agricultural production; electricity production, telecommunications, and transport also contributed to growth. Public and private investment grew

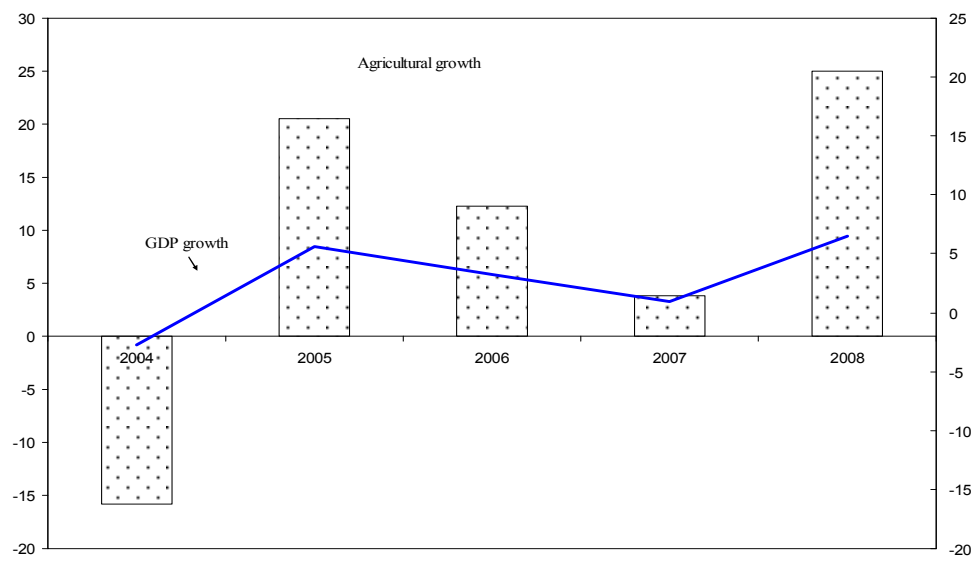
strongly, reflecting expansion in mining, telecommunications, transport equipment, and road construction; the investment/GDP ratio rose from 23.6 percent in 2007 to 26.4 percent in 2008. From 2000, with the restoration of political stability, average GDP growth in Niger has been higher than in the WAEMU zone as a whole, although lower than in Sub-Saharan Africa.

\begin{tabular}{|c|c|c|c|c|c|c|c|c|c|c|}
\hline \multicolumn{11}{|c|}{$\begin{array}{l}\text { On average, Niger tends to grow faster than WAEMU but slower than Sub-Saharan Africa } \\
\text { (Rates of growth, 2000-2008) }\end{array}$} \\
\hline & 2000 & 2001 & 2002 & 2003 & 2004 & 2005 & 2006 & 2007 & 2008 & Average \\
\hline Niger, GDP growth & -2.5 & 7.4 & 5.3 & 7.1 & -0.8 & 8.4 & 5.8 & 3.3 & 9.5 & 4.8 \\
\hline Benin & 4.9 & 6.2 & 4.5 & 3.9 & 3.1 & 2.9 & 3.8 & 4.6 & 5.1 & 4.3 \\
\hline Burkina Faso & 1.8 & 6.6 & 4.7 & 7.3 & 4.6 & 7.1 & 5.5 & 3.6 & 4.5 & 5.1 \\
\hline Cote D'Ivore & -4.6 & 0.0 & -1.6 & -1.7 & 1.6 & 1.9 & 0.7 & 1.6 & 2.9 & 0.1 \\
\hline Guinea-Bissau & 7.5 & 0.2 & -7.1 & -0.6 & 2.2 & 3.2 & 1.8 & 2.5 & 3.2 & 1.4 \\
\hline Mali & -3.2 & 12.1 & 4.3 & 7.2 & 2.4 & 6.1 & 5.3 & 3.1 & 4.8 & 4.7 \\
\hline Togo & -1.3 & -2.3 & -0.3 & 5.2 & 2.4 & 1.3 & 4.1 & 2.1 & 2.5 & 1.5 \\
\hline WAEMU, GDP growth & -0.9 & 4.2 & 1.6 & 3.8 & 2.9 & 4.5 & 3.2 & 3.2 & 4.1 & 3.0 \\
\hline Sub-Saharan Africa, GDP growth & 3.6 & 5.0 & 7.0 & 5.0 & 7.0 & 6.3 & 6.3 & 6.6 & 5.3 & 5.8 \\
\hline
\end{tabular}

\section{Because of the likelihood that agricultural production may fall somewhat} short of the record 2008 level, GDP growth for 2009 has been revised downwards to 3 percent. However, non-agricultural growth should continue to be strong, at about 5.2 percent, despite the international downturn, because of foreign-financed investment in mining, petroleum, dam and road construction, which have been recently launched, and further expansion in electricity, construction, and transport. In mining, a French company has signed an agreement with the government in January 2009 to develop the large uranium Imouararen field which would almost triple uranium production by 2015 , and investment is also picking up to upgrade existing mines. In petroleum, a production sharing agreement was signed in June 2008 with China's National Oil and Gas Exploration and Development Corporation (CNODC) involving the development of a large oil field, the construction of a 


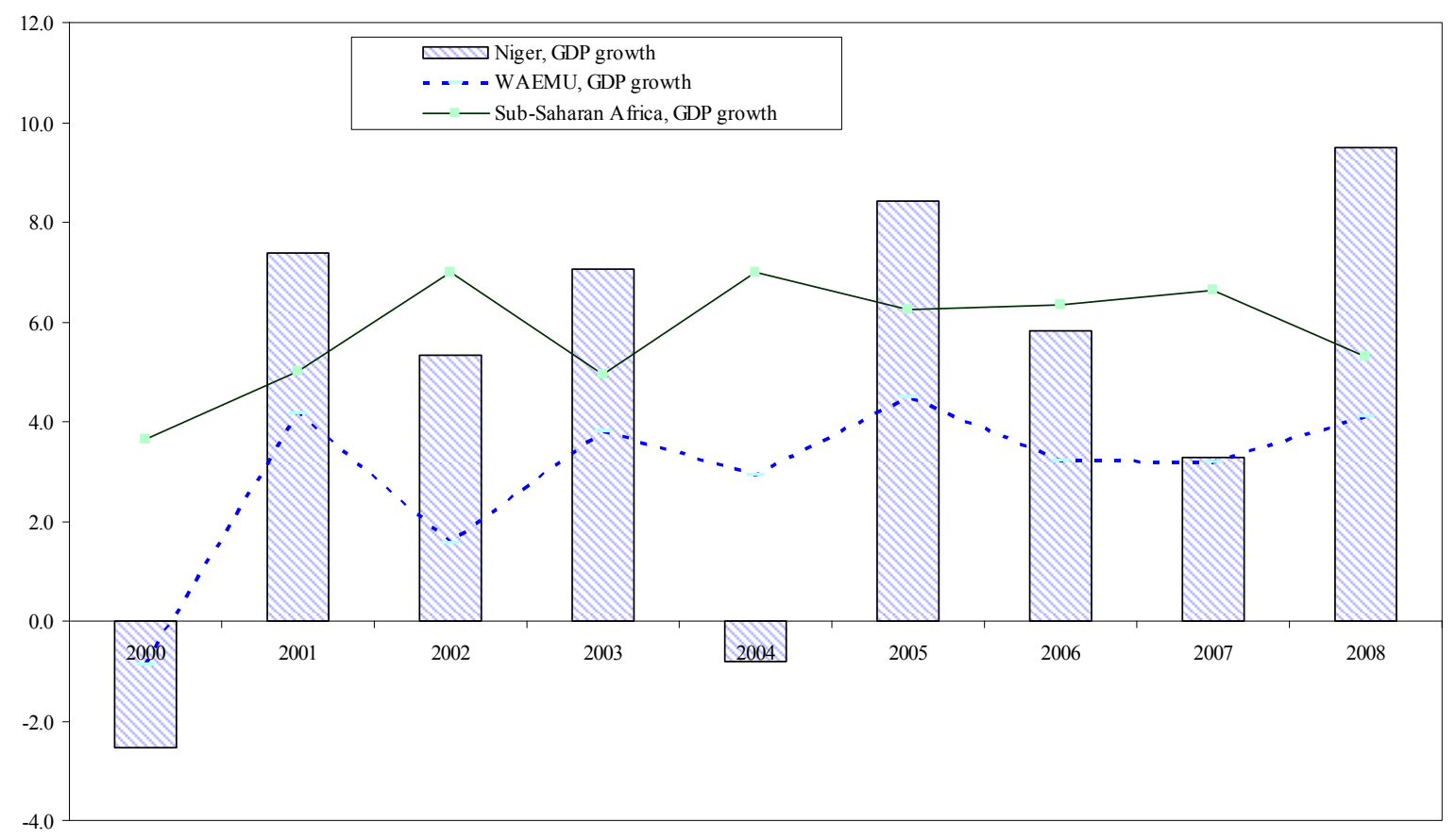

pipeline and of a refinery in Zinder, close to the Nigerian border with the oil production starting in 2012. The drilling program is very ambitious, with 100 wells to be drilled over a three-year period. The financing of the Kandaji dam on the river Niger has been finalized with a number of multilateral lending agencies, but work is expected to start only toward the end of the year; the dam would greatly increase the irrigation potential in the Niger river. In addition, the government has started a major program of expanding electricity production, in order to have back-up capacity in case Nigeria cannot export electricity through the existing interconnected transmission system.

\section{Inflation accelerated in} the first half of 2008, reaching a peak of 15 percent in August; it declined thereafter to 10.6 percent in the 12-months to February 2009, as prices of petroleum products, transportation and a number of food stuff declined. However, as elsewhere in the region, cereals prices remained seasonally high in recent months, despite the excellent harvest. This may reflect the only moderate decline

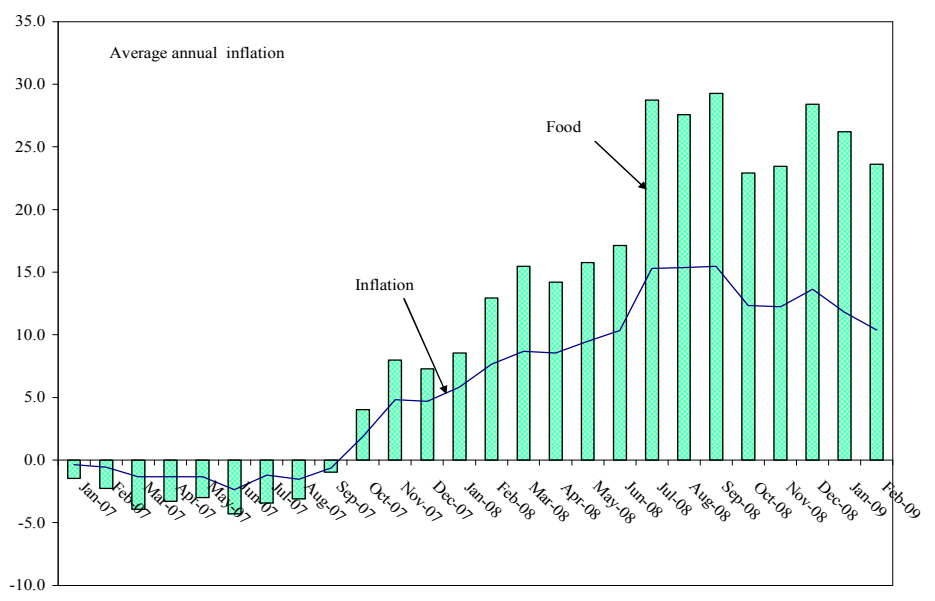


of international cereal prices, and also the higher income to farmers from bumper crops of beans and vegetables, which allow producers to retain cereals for sale in the lean season.

\section{In 2008, the balance of payments current account deficit increased to}

\section{4 percent of GDP, from}

10 percent in 2007 . This reflected

mainly the increase in import of capital goods and the price increase for petroleum and imported food. FDI rose significantly, associated with investment in mining. Official reserves attributed to Niger rose during the year, as a result of the large petroleum signature bonus received in June (5.4 percent of GDP).

5. Credit to the economy

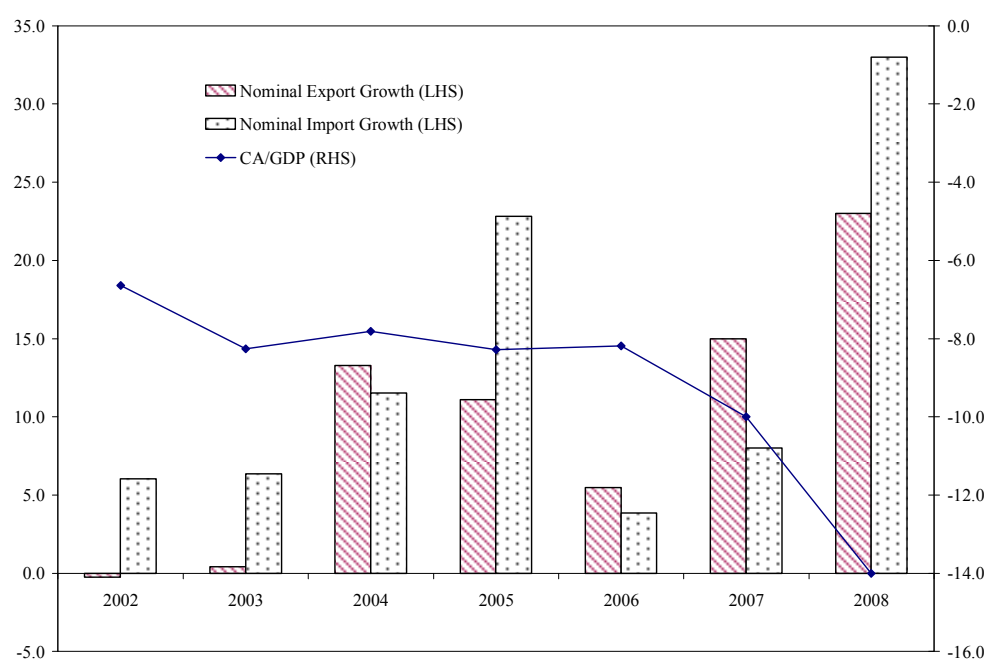
continued to be buoyant, after

few years of fast growth, albeit from a low base, and rose by 37 percent in 2008 reflecting loans to the mining, telecommunication, transportation, and commerce sectors. With credit growth exceeding that of deposits, banks drew down a part of their liquid deposits at the central bank, which were still at a comfortable level by end-year-at 14 percent of deposits; they also made use of external medium-term credit lines, extended by the European Investment Bank and other agencies. Nonperforming loans, after having risen in recent years, declined during 2008 by 5 percentage points, to 16.5 percent of gross loans, and are mostly provisioned.

6. The present level of the real effective exchange rate appears consistent with fundamentals. Its real appreciation of 23.8 percent since 2001 is consistent with macroequilibrium, in view of the favorable movement of Niger's terms of trade in recent years, reflecting higher prices for uranium. Moreover, projected increases in foreign direct investment, and the prospects for petroleum production are likely to lead to a further appreciation of the equilibrium real exchange rate in the next few years.

7. The fiscal performance was strong in $\mathbf{2 0 0 8}$ and all quantitative and structural performance criteria for end-2008 were met (Tables $2 \mathrm{a}$ and $2 \mathrm{~b}$, and Appendix Table 1a). Tax revenue exceeded program targets by 0.9 percent of GDP, reaching 11.8 percent of GDP because of strong performance of custom duties, company profit taxes and the VAT. This result is even more noteworthy as the strong GDP growth resulted from the agricultural sector which is lightly taxed. Nontax revenue also exceeded projections, mainly because of higher interest on central bank deposits. On the expenditure side, both current and domestically-financed capital expenditure remained below program targets, for a total of 
1 percent of GDP, partly because of deferment to 2009 of the government contribution to the refinery, and also because of delays in implementing the mid-2008 supplementary budget. As a result, the basic balance, which excludes foreign-financed expenditure, was stronger than expected by about 2 percent of GDP, and government accumulation of deposits at the central bank was much higher than targeted. Budgetary data show that expenditure in the social and rural sectors rose significantly as a share of GDP in 2008 (Table 5), as the supplementary budget appropriately focused on the priority sectors.

Fiscal Performance in 2008 was better than programmed

\begin{tabular}{|c|c|c|c|c|}
\hline & \multicolumn{2}{|c|}{2008} & \multicolumn{2}{|c|}{2009} \\
\hline & Prog. & Prel. Est. & Init. Prog & Rev. Prog \\
\hline & \multicolumn{4}{|c|}{ (in percent of GDP) } \\
\hline Revenue & 17.5 & 18.4 & 12.2 & 12.2 \\
\hline Tax revenue & 11 & 11.7 & 11.5 & 11.7 \\
\hline Nontax revenue & 6.4 & 6.6 & 0.6 & 0.4 \\
\hline Of which: exceptional mining receipts & 5.3 & 5.1 & 0 & 0 \\
\hline Total Expenditure & 24.2 & 22.9 & 26.0 & 24.6 \\
\hline Of which: domestically financed & 18.1 & 17.1 & 17.5 & 18.0 \\
\hline Current expenditures & 12.9 & 12.5 & 12.7 & 12.3 \\
\hline Capital expenditures & 11.3 & 10.3 & 13.3 & 12.3 \\
\hline Of wlhich: domestically financed & 4.6 & 4.0 & 4.3 & 5.2 \\
\hline Overall balance & -6.7 & -4.4 & -13.8 & -12.4 \\
\hline Basic balance & -0.6 & 1.3 & -5.3 & -5.8 \\
\hline
\end{tabular}

Sources: Nigerien authorities; and IMF staff estimates.

8. The 2007 PRSP Annual Progress Report issued in late 2008 indicates that a number of key social indicators have improved; in particular, infant and maternal health coverage rose, and utilization of health facilities increased, following the adoption in 2007 of the policy of free access to health care for children under 5 and pregnant women (Tables 6 and 7). The 2008 PRSP Annual Progress Report is to be completed in July 2009, so as to serve as an input to the 2010 budget preparation.

9. Results of the recent household survey indicate that incidence of poverty declined from 62 percent in 2005 to 59.5 percent in 2007/08; poverty depth indicators also show an improvement. The decline in poverty has been stronger in urban than in rural areas.

\section{Program Discussions}

10. The discussions focused on the macroeconomic prospects for 2009, the budgetary outlook, the progress in structural reforms, notably in the fiscal and financial sectors, and management issues in the petroleum and mining sectors (LOI paragraphs 8-18). 
The key challenge is to sustain growth with macrostability

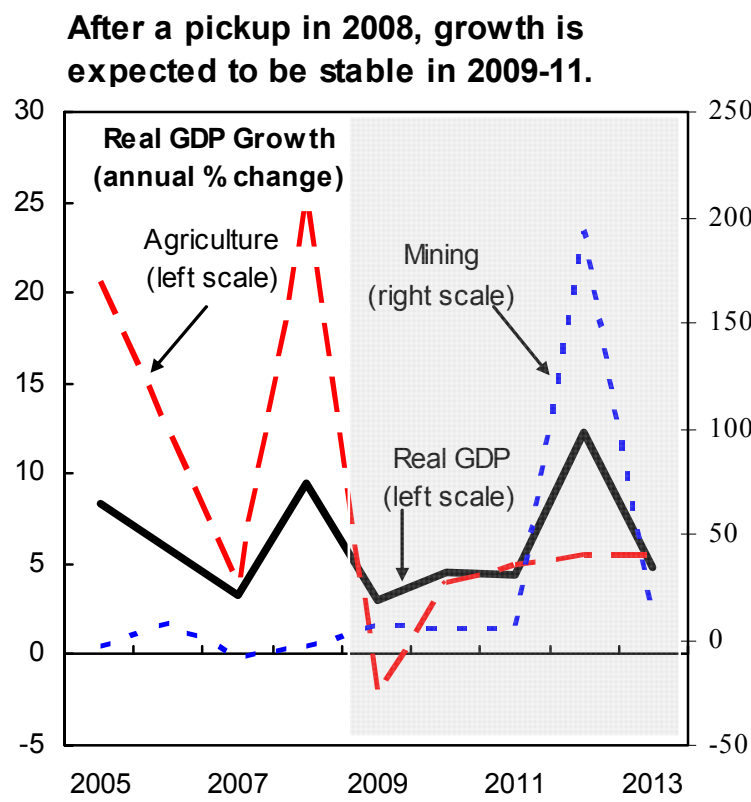

\author{
Inflation is decelerating.
}

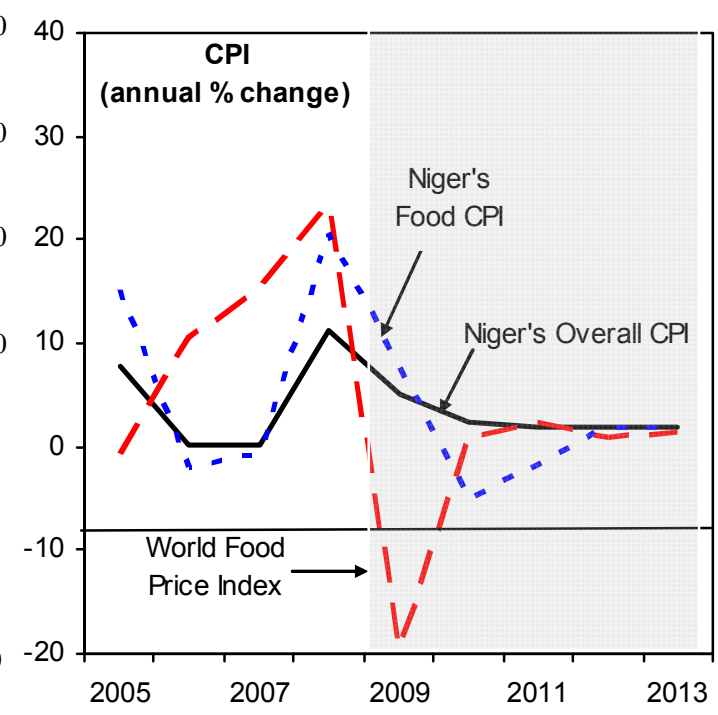

FDI will finance a large current account deficit in 2009-11...

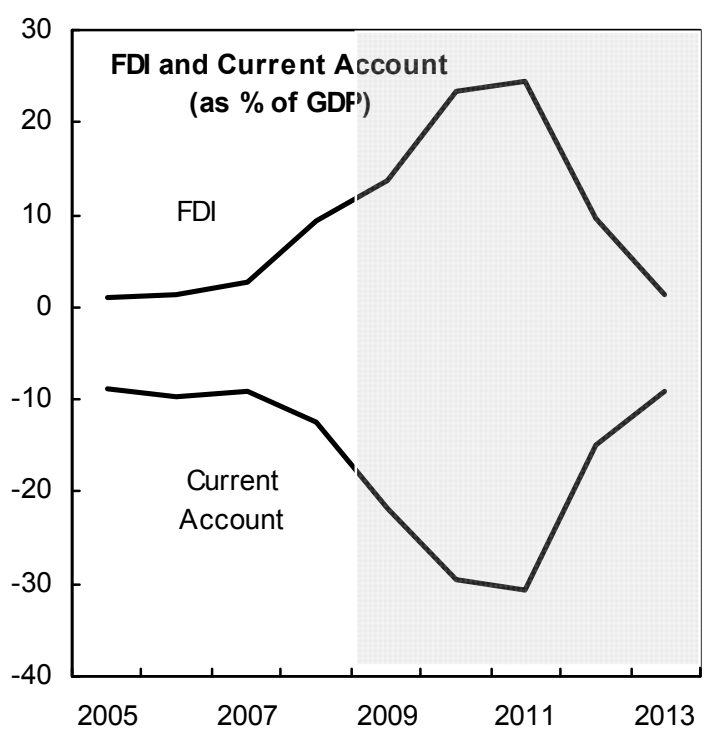

...while the terms of trade are expected to improve in 2009-11.

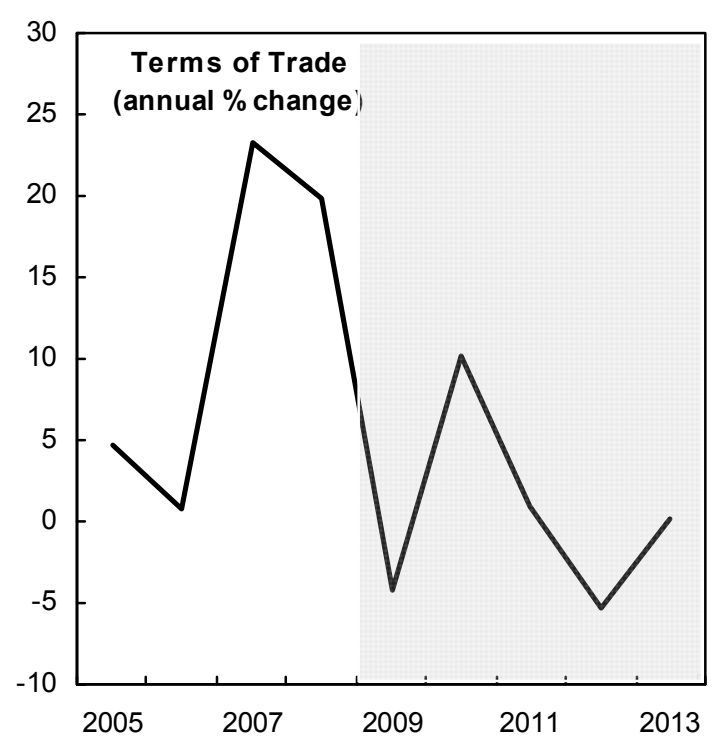

Source: Nigerien authorities and IMF staff estimates and projections. 
The fiscal position is sound.

Exceptional non-tax revenue in 2008 and increased tax revenue...

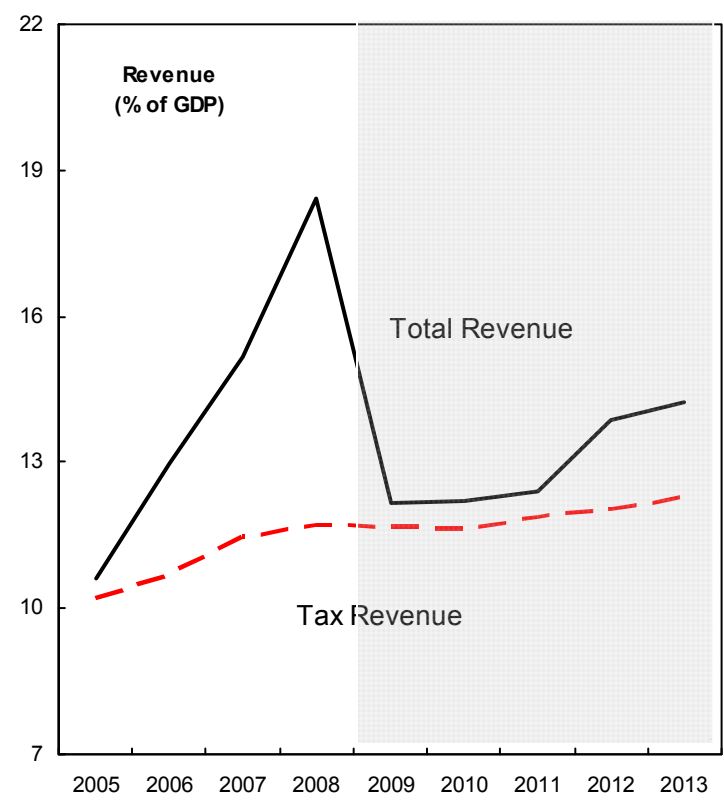

...allow for increased spending...

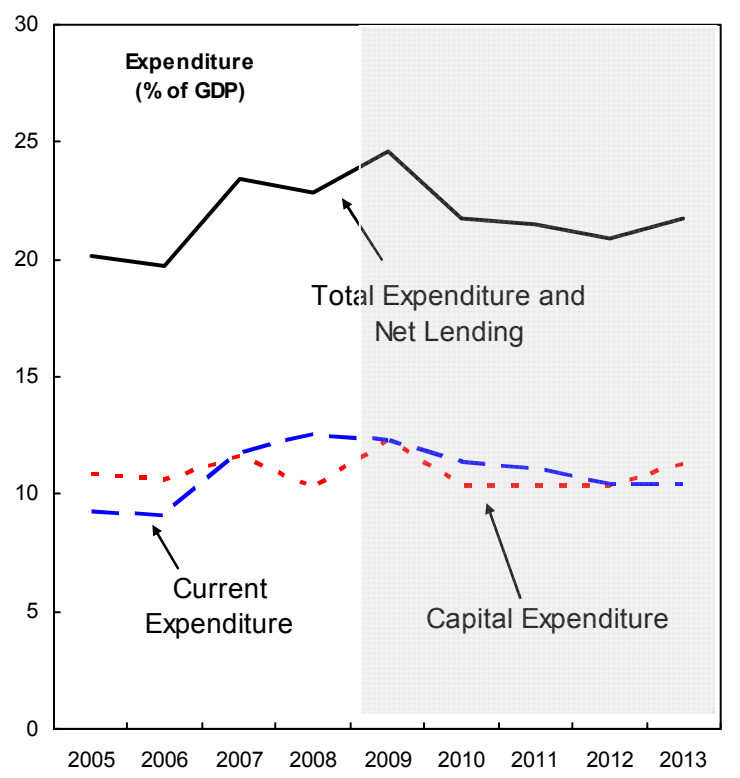

...together with external support...

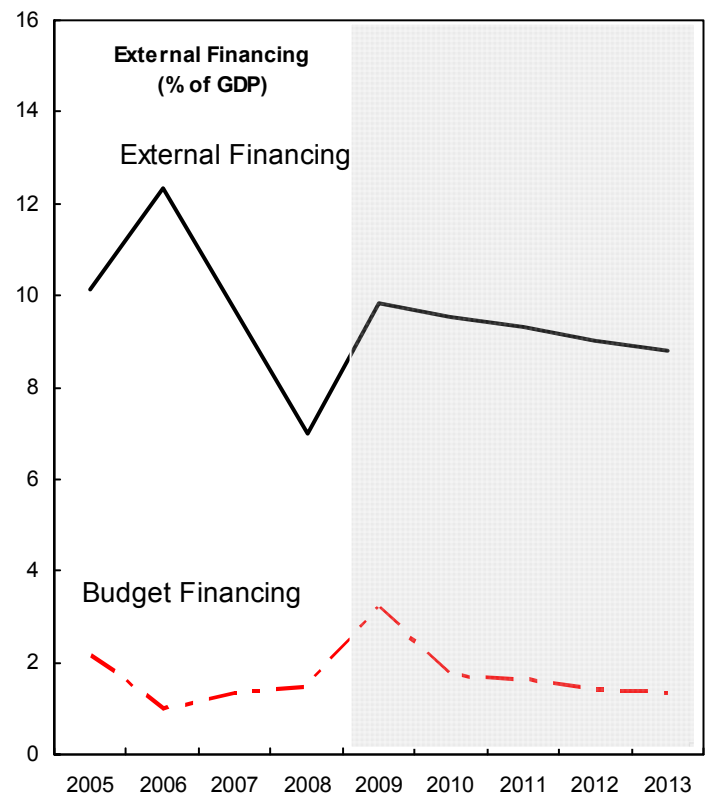

...while the widening deficit in 2009 is expected to be temporary. ${ }^{1 /}$

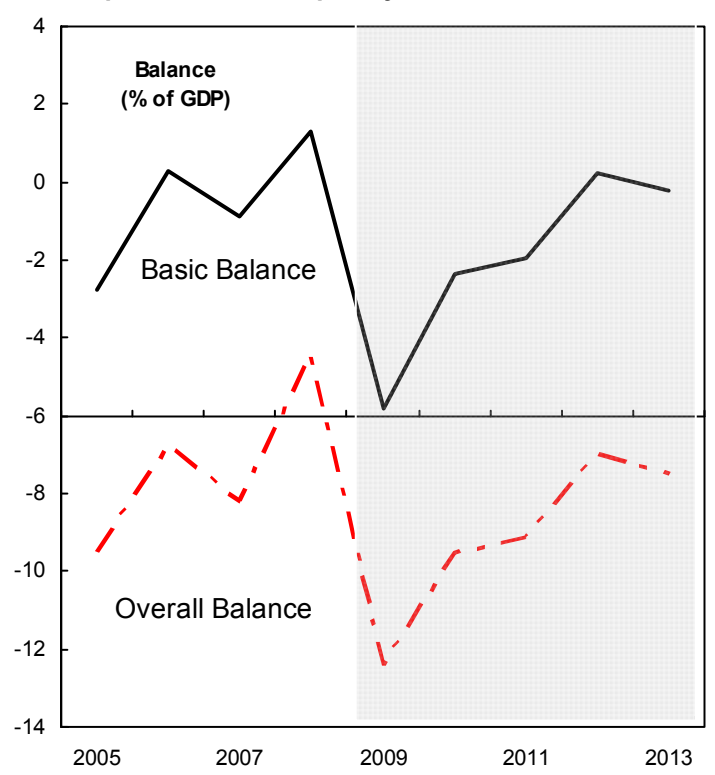

Source: Nigerien authorities and IMF staff estimates and projections.

1/ A large signature bonus for a petroleum production sharing contract was received in 2008 and will be spent in 2009. 
External debt is sustainable...

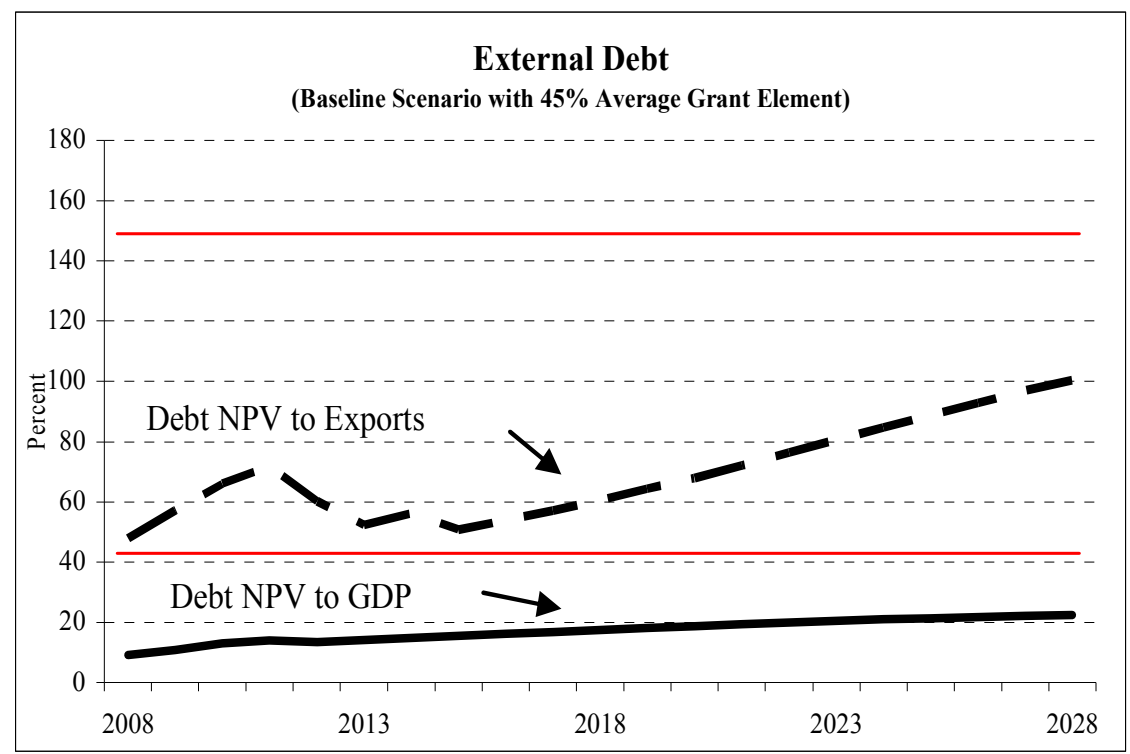

...but remains sensitive to borrowing terms.

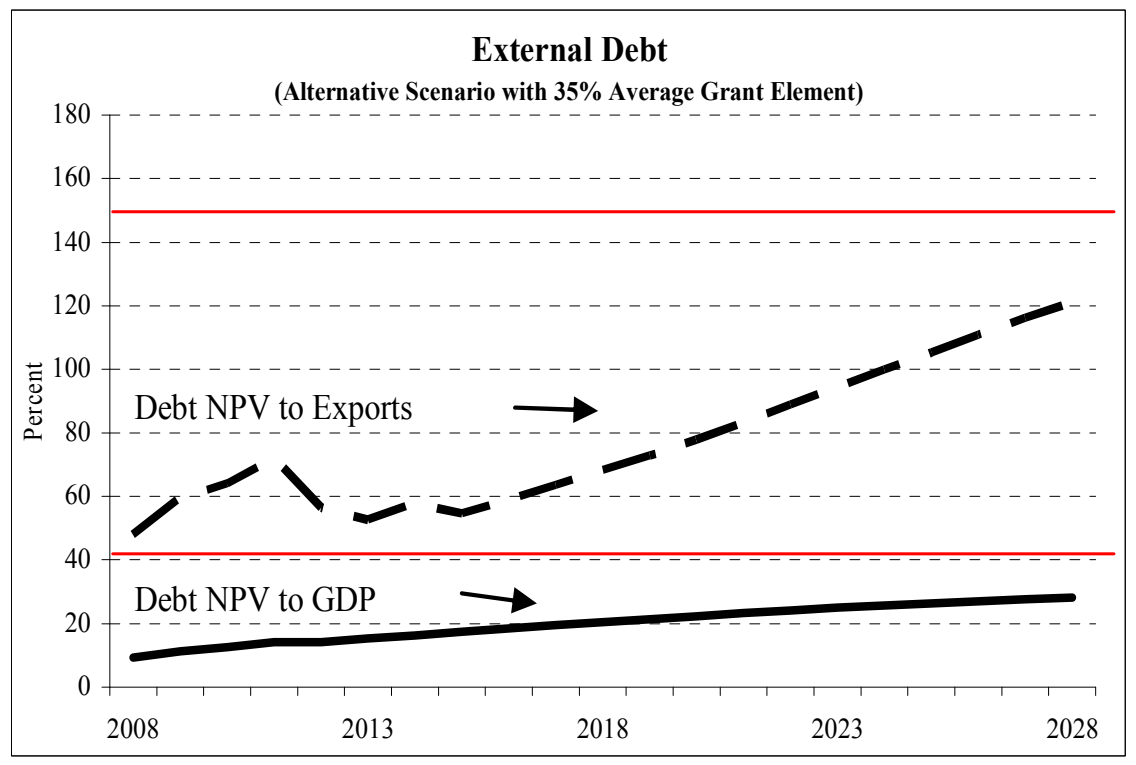

Source: IMF staff estimates and projections. 


\section{A. Macroeconomic Framework and Risk to the Outlook}

11. As indicated above, growth prospects are positive for 2009, because of large private and public investments. The investment ratio is projected to rise to 36 percent of GDP, on the strength of ongoing private investment plans in uranium mining, petroleum exploration and the refinery, and public investment on road building, the initiation of the Kandaji dam project, and expansion of electricity production capacity. The external current account deficit is projected to rise to 24 percent of GDP, as capital goods imports increase, financed mostly by foreign direct investment. Growth of credit is expected to decelerate, as banks are gradually reducing their liquidity buffer at the central bank. The rate of inflation on an end-year basis is projected to fall to about 2 percent despite an expansionary budgetary stance; since the increase in government spending is focused on strengthening infrastructure, with a large import content, its impact on inflation should be subdued.

12. The main risk to the outlook is given by the climatic conditions which could adversely affect agricultural production. It is therefore important that the food security organizational arrangements be further strengthened, in close consultation with donors. Downside risks from the international recession are modest, because of the ongoing large investment projects, the long-term export contracts for minerals, and the regional market destination of exports of livestock and foodstuff.

\section{B. Budgetary Outlook for 2009 and Fiscal Reforms}

\section{After the strong performance in 2008, revenue targets for 2009 have been} moved slightly up to 11.7 percent of GDP, from 11.5 in the original program. On the expenditure side, capital outlays have been raised by the equivalent of 1 percent of GDP to reflect the spending for the refinery, originally envisaged for 2008; this is partly offset by lower outlays on subsidies and transfers. In all, the basic budgetary deficit is projected to be 0.5 percent of GDP higher than under the original program, and is reflected in higher domestic financing, through the draw-down of deposits at the central bank. These have been boosted over the last two years by exceptional mining revenues, but would still remain by end-2009 at a comfortable level, equivalent to about 3 percent of GDP. Budgetary aid projections for 2009 remain very close to initial forecasts, and represent a significant increase from 2008, reflecting carry forward of disbursements originally planned for 2008, and stronger support to the crucial education sector. ${ }^{1}$ Foreign-funded public investments have been scaled down from the original forecast, based on realistic execution levels.

\footnotetext{
${ }^{1}$ Donors support to a common fund for education is slated to double in 2009 to about CFAF 5 billion.
} 


\section{The authorities are continuing comprehensive PFM reforms (LOI}

paragraphs 10-11). These focus on (i) strengthening the financial comptrollers in the various ministries, and in the regions; (ii) the computerization of decentralized budgetary offices; and (iii) the preparation of a medium-term expenditure framework, in close consultation with the PRSP secretariat, which should underpin the budget for 2010. Procedures to permit full refunds of VAT credits to exporters are to be finalized by end-year, together with a reform of real estate and fixed assets taxation, to exclude industrial equipment. The company profit tax is scheduled to be cut from 35 percent to 30 percent in the 2010 budget year, to conform with similar reductions in the region.

\section{Financial Sector Reforms and Mining Management Issues}

\section{Important reforms are under way in the financial sector (LOI paragraphs 13}

and 16). All banks are making preparations to meet the new higher capital requirement of CFAF 5 billion which enters into effect at end-2010, with two out of 10 banks already meeting it. The new postal bank Finaposte, which aims at mobilizing savings and improving the payment system, is expected to obtain the banking licence from the regional banking commission in its June session, after the government will have paid in the required equity capital; the staff reiterated its recommendation that the postal bank should refrain from lending operations. While the government is determined to go ahead with the establishment of an agricultural bank to increase farmers' access to credit, authorities concur with the staff view that the operational aspects will have to be prepared with utmost care to minimize the risk of operational losses and fiscal costs that similar banks have often incurred in the region.

16. With the ongoing construction of a refinery in Zinder, to process the petroleum which will be brought by pipeline from the Agadem field, it is important that arrangements for the export to neighbouring countries of the large share of the output exceeding local consumption be finalized well before the refinery enters into operation in $2012 .^{2}$ Staff also underscored the importance that the government budget for the coming years include the 5 percent government share of drilling costs in the Agadem field, which could amount to about US\$25 million per year (see LOI paragraphs 17-18); similarly, the government share of the development costs of the Azelik uranium mine which will become operational next year will have to be budgeted transparently (LOI para.19). The government participation in these operations is consistent with the mining and petroleum laws, which provide for a minimum ownership share that the operators must grant to the government on request, with the corresponding investment costs to be recovered from future profits; ownership shares above the threshold give rise to immediate cost sharing.

\footnotetext{
${ }^{2}$ The refinery will produce annually 7 billion barrels of petroleum products, while total national consumption is about one third of this.
} 


\section{Program Monitoring ANd Amendments}

17. In the attached LOI, the authorities request the modification of the domestic budgetary financing performance criterion for end-June and end-December 2009, to reflect new data on aid disbursement in the first half of the year, and higher capital expenditure (Table 1b of the LOI). Following the recent Board decision on conditionality, it is proposed that the structural performance criteria for end-2009 on the elimination of the ceiling for reimbursing VAT credits, and the reduction of the profit tax from 35 to 30 percent, be converted into benchmarks; their observance will be an important element of the fourth review under the arrangement.

\section{STAFF APPRAISAL}

18. Niger has recorded strong growth in 2008 because of an excellent harvest and important investments in mining, and infrastructure. The prospects for growth in 2009 remain favourable, despite the international downturn, in view of the recently agreed private projects in mining, and the ongoing expansion of public infrastructure investment. It is encouraging that efforts to improve productivity in agriculture, the mainstay of the economy, are beginning to bear fruit.

19. The good results achieved in $\mathbf{2 0 0 8}$ in tax mobilization are encouraging, and reflect the ongoing efforts to improve tax administration. The authorities must also be commended for improved controls on procurement procedures. It is important to further improve, as planned, expenditure controls and management, including better monitoring of decentralized outlays, and to strengthen the medium-term expenditure framework, so as to prepare the next year budget within a medium-term context and with close alignment to PRSP priorities.

20. The authorities must remain vigilant in their debt management policy. Limiting public sector risk also calls for keeping the public sector participation in mining projects at modest level.

21. The progress in reforming the financial sector is encouraging, and the ongoing recapitalization of banks, in line with the regional banking commission guidelines, is welcome. The financial system remains hampered by the paucity of long-term resources, and any additional mobilization of such resources from international agencies would be welcome.

22. In light of the satisfactory performance so far, and observance of the end-December 2008 performance criteria and continuous performance criteria, the staff recommends completion of the review. It also recommends the approval of the proposed modification of performance criteria, including the modification of the quantitative performance criterion on domestic budgetary financing for end-June and end-December 2009, and the conversion of two structural performance criteria for end-2009 into structural benchmarks, following the recent change of Fund policy on this matter. 
Table 1. Niger: Selected Economic and Financial Indicators, 2006-11

\begin{tabular}{|c|c|c|c|c|c|c|c|c|}
\hline & \multirow{3}{*}{2006} & \multirow{3}{*}{$\begin{array}{c}2007 \\
\text { Est. }\end{array}$} & \multirow{2}{*}{\multicolumn{2}{|c|}{2008}} & \multirow{2}{*}{\multicolumn{2}{|c|}{2009}} & & \\
\hline & & & & & & & \multirow{2}{*}{$\begin{array}{c}2010 \\
\text { Projections }\end{array}$} & \multirow[t]{2}{*}{2011} \\
\hline & & & 1st Rev. & Est. & 1st Rev. & Projections & & \\
\hline & \multicolumn{8}{|c|}{ (Annual percentage change, unless otherwise indicated) } \\
\hline \multicolumn{9}{|l|}{ National income and prices } \\
\hline GDP at constant prices & 5.8 & 3.3 & 5.9 & 9.5 & 4.5 & 3.0 & 4.5 & 4.5 \\
\hline GDP deflator & 1.4 & 3.3 & 8.3 & 7.6 & 2.2 & 3.8 & 2.6 & 1.9 \\
\hline \multicolumn{9}{|l|}{ Consumer price index } \\
\hline Annual average & 0.1 & 0.1 & 9.0 & 11.3 & 2.5 & 5.0 & 2.3 & 2.0 \\
\hline End of period & 0.4 & 0.4 & 8.5 & 13.6 & 2.0 & 2.0 & 2.0 & 2.0 \\
\hline \multicolumn{9}{|l|}{ External sector } \\
\hline Exports, f.o.b. (CFA francs) & 5.4 & 19.8 & 15.3 & 25.0 & 6.5 & -2.0 & 15.4 & 3.0 \\
\hline Of which: non-uranium exports & 7.3 & -5.8 & 8.6 & 12.4 & 5.3 & 2.4 & 9.7 & 6.0 \\
\hline Imports, f.o.b (CFA francs) & -3.6 & 9.7 & 20.7 & 33.2 & 60.3 & 30.9 & 26.3 & 8.8 \\
\hline Export volume & 2.4 & -4.2 & 0.0 & -2.2 & 7.5 & 5.7 & 6.4 & 2.9 \\
\hline Import volume & -1.5 & 7.8 & 13.8 & 25.1 & 64.8 & 38.6 & 24.3 & 7.0 \\
\hline Terms of trade (deterioration -) & 0.8 & 23.3 & 9.0 & 19.9 & 2.1 & -3.3 & 6.6 & -1.6 \\
\hline Nominal effective exchange rate (depreciation -) & 3.6 & 2.6 & $\ldots$ & 0.8 & $\ldots$ & $\ldots$ & $\ldots$ & $\ldots$ \\
\hline Real effective exchange rate (depreciation -) & 1.5 & 3.9 & $\ldots$ & 9.8 & $\ldots$ & $\ldots$ & $\ldots$ & $\ldots$ \\
\hline \multicolumn{9}{|l|}{ Government finances } \\
\hline Total revenue & 30.8 & 25.0 & 31.8 & 43.0 & -25.2 & -29.3 & 7.1 & 8.5 \\
\hline Total expenditure and net lending ${ }^{1}$ & 5.1 & 26.4 & 18.6 & 15.1 & 15.5 & 15.1 & -6.0 & 5.2 \\
\hline Of which: current expenditure ${ }^{2}$ & 5.4 & 37.4 & 25.8 & 25.7 & 5.7 & 5.0 & -0.8 & 4.1 \\
\hline capital expenditure & 4.8 & 16.9 & 11.3 & 4.4 & 26.6 & 27.4 & -11.1 & 6.4 \\
\hline & \multicolumn{8}{|c|}{ (Annual change as percent of beginning-of-period broad money) } \\
\hline \multicolumn{9}{|l|}{ Money and credit } \\
\hline Domestic credit ${ }^{3}$ & -16.1 & -3.5 & 4.5 & 1.8 & 22.6 & 30.5 & 12.6 & 10.5 \\
\hline Credit to the government (net) ${ }^{3}$ & -31.6 & -14.7 & -1.6 & -18.1 & 13.8 & 21.3 & 2.4 & 0.5 \\
\hline Credit to the economy ${ }^{3}$ & 15.4 & 11.2 & 6.1 & 19.9 & 8.9 & 9.2 & 10.2 & 10.0 \\
\hline Net domestic assets ${ }^{3}$ & -17.3 & -0.9 & 4.5 & -2.9 & 22.6 & 28.0 & 12.0 & 10.5 \\
\hline Broad Money & 16.2 & 23.0 & 24.1 & 12.7 & 18.7 & 23.8 & 15.4 & 13.8 \\
\hline Velocity of broad money (in percent) & 6.6 & 5.7 & 5.3 & 6.0 & 4.8 & 5.2 & 4.8 & 4.5 \\
\hline & \multicolumn{8}{|c|}{ (Percent of GDP, unless otherwise indicated) } \\
\hline Government finances & & & & & & & & \\
\hline Total revenue & 13.0 & 15.2 & 17.5 & 18.4 & 12.2 & 12.2 & 12.2 & 12.4 \\
\hline Of which: exceptional mining receipts & 1.6 & 1.4 & 5.3 & 5.1 & $\ldots$ & $\ldots$ & $\ldots$ & $\ldots$ \\
\hline Total expenditure and net lending & 19.8 & 23.4 & 24.2 & 22.9 & 26.0 & 24.6 & 21.6 & 21.3 \\
\hline Current expenditure $^{2}$ & 9.1 & 11.8 & 12.9 & 12.5 & 12.7 & 12.3 & 11.4 & 11.1 \\
\hline Capital expenditure & 10.6 & 11.6 & 11.3 & 10.3 & 13.3 & 12.3 & 10.2 & 10.2 \\
\hline Basic balance (excluding grants) ${ }^{4}$ & 0.30 & -0.9 & -0.6 & 1.29 & -5.3 & -5.8 & -2.2 & -1.7 \\
\hline Overall balance (commitment basis, excluding grants) & -6.8 & -8.2 & -6.7 & -4.4 & -13.8 & -12.4 & -9.4 & -8.9 \\
\hline Overall balance (commitment basis, including grants) & -0.8 & -1.0 & -0.47 & 1.50 & -4.5 & -4.4 & -3.0 & -2.6 \\
\hline Gross investment & 23.6 & 23.6 & 26.3 & 26.4 & 36.6 & 36.1 & 45.2 & 49.0 \\
\hline Of which: non-government investment & 16.8 & 17.2 & 19.4 & 19.8 & 28.6 & 28.7 & 39.0 & 42.9 \\
\hline government & 6.8 & 6.4 & 6.8 & 6.7 & 8.0 & 7.4 & 6.2 & 6.1 \\
\hline Gross national savings & 13.9 & 14.6 & 16.4 & 13.9 & 14.0 & 14.9 & 14.9 & 17.0 \\
\hline Of which: non-government & 10.0 & 11.2 & 11.9 & 8.0 & 9.3 & 8.8 & 7.9 & 9.7 \\
\hline Domestic savings & 10.5 & 11.4 & 12.6 & 11.2 & 7.9 & 11.6 & 14.1 & 16.3 \\
\hline External current account balance & & & & & & & & \\
\hline Excluding official grants & -10.9 & -10.0 & -12.3 & -13.6 & -26.9 & -24.0 & -31.5 & -33.2 \\
\hline Including official grants & -9.7 & -9.0 & -9.9 & -12.6 & -22.5 & -21.2 & -30.3 & -32.0 \\
\hline Debt-service ratio as percent of: & & & & & & & & \\
\hline Exports of goods and services ${ }^{5}$ & 252.8 & 2.9 & 2.8 & 2.8 & 2.8 & 3.0 & 3.0 & 3.8 \\
\hline Government revenue $^{5}$ & 320.1 & 3.4 & 3.1 & 2.8 & 4.4 & 4.2 & 4.5 & 5.6 \\
\hline NPV of external debt & 10.2 & 10.5 & 9.2 & 9.2 & 10.9 & 10.9 & 11.8 & 12.7 \\
\hline Foreign Aid & 9.1 & 9.8 & 7.8 & 7.2 & 12.3 & 10.2 & 9.7 & 9.5 \\
\hline & & & & (Billions $\mathrm{Cl}$ & ancs) & & & \\
\hline GDP at current market prices & 1,906 & 2,035 & 2,333 & 2,398 & 2,508 & 2,564 & 2,748 & 2,927 \\
\hline Overall balance of payments & 98.2 & 68.9 & 96.5 & 55.5 & -15.2 & -9.0 & 18.5 & 21.0 \\
\hline
\end{tabular}

Sources: Nigerien authorities; and IMF staff estimates and projections.

${ }^{1}$ Commitment basis per payment orders issued.

${ }^{2}$ Including budget reserve

${ }^{3}$ Percent of beginning-of-period broad money stock.

${ }^{4}$ Total revenue, excluding grants, minus total expenditure, excluding foreign-financed investment projects

${ }^{5}$ After HIPC and MDRI debt relief starting in 2006. 
Table 2a. Niger: Financial Operations of the Central Government, 2006-11

\begin{tabular}{|c|c|c|c|c|c|c|c|c|}
\hline & \multirow{2}{*}{$\frac{2006}{\text { Est. }}$} & \multirow{2}{*}{$\begin{array}{r}2007 \\
\text { Est. }\end{array}$} & \multicolumn{2}{|c|}{2008} & \multicolumn{2}{|c|}{2009} & 2010 & 2011 \\
\hline & & & 1st Rev. & Est. & 1st Rev. & Proj. & \multicolumn{2}{|c|}{ Projections } \\
\hline & \multicolumn{8}{|c|}{ (Billions of CFA francs) } \\
\hline Total revenue & 247.2 & 309.0 & 407.4 & 442.0 & 304.8 & 312.3 & 334.6 & 363.1 \\
\hline Tax revenue & 203.8 & 233.2 & 255.8 & 281.1 & 288.4 & 299.3 & 319.5 & 347.8 \\
\hline Of which: International trade & 95.8 & 100.7 & 100.7 & 110.7 & 105.9 & 107.5 & 116.9 & 127.0 \\
\hline Nontax revenue & 38.5 & 71.2 & 149.0 & 158.8 & 14.1 & 10.1 & 12.0 & 12.0 \\
\hline Of which: exceptional uranium receipts & 30.4 & 27.5 & 123.4 & 123.4 & 0.0 & 0.0 & 0.0 & 0.0 \\
\hline Special accounts revenue & 4.9 & 4.6 & 2.6 & 2.1 & 2.3 & 2.9 & 3.1 & 3.3 \\
\hline Total expenditure and net lending & 376.6 & 476.0 & 564.5 & 547.9 & 651.7 & 630.8 & 593.1 & 623.9 \\
\hline Of which: domestically financed & 241.4 & 326.9 & 422.3 & 411.1 & 438.4 & 460.8 & 396.2 & 414.1 \\
\hline Total current expenditure & 174.2 & 239.4 & 301.2 & 300.8 & 318.3 & 316.0 & 313.3 & 326.1 \\
\hline Budgetary expenditure & 163.8 & 199.3 & 278.5 & 264.1 & 292.9 & 285.6 & 298.2 & 310.0 \\
\hline Wages and salaries & 68.0 & 72.2 & 86.3 & 83.8 & 93.3 & 93.3 & 99.8 & 106.8 \\
\hline Materials and supplies & 47.4 & 61.7 & 71.5 & 63.4 & 88.2 & 87.6 & 89.6 & 91.4 \\
\hline Subsidies and transfers & 43.5 & 58.3 & 105.3 & 102.1 & 104.8 & 98.4 & 100.7 & 102.7 \\
\hline of which: Transfers to other levels of government & $\ldots$ & $\ldots$ & 7.1 & 7.5 & 0.0 & 0.0 & 0.0 & 0.0 \\
\hline Interest, scheduled & 4.9 & 7.1 & 7.4 & 5.5 & 6.6 & 6.3 & 8.1 & 9.1 \\
\hline Of which: External debt & 3.7 & 4.4 & 4.5 & 3.7 & 4.9 & 4.5 & 6.3 & 7.2 \\
\hline Adjustments and fiscal expenditures & 0.0 & 0.3 & 8.0 & 9.3 & 0.0 & 0.0 & 0.0 & 0.0 \\
\hline Special accounts expenditure & 10.4 & 39.8 & 22.7 & 36.7 & 25.4 & 30.4 & 15.1 & 16.2 \\
\hline Capital expenditure and net lending & 202.3 & 236.6 & 263.3 & 247.1 & 333.4 & 314.8 & 279.8 & 297.7 \\
\hline Capital expenditure & 202.3 & 236.6 & 263.3 & 247.1 & 333.4 & 314.8 & 279.8 & 297.7 \\
\hline Domestically financed & 51.5 & 73.7 & 107.5 & 96.7 & 107.7 & 132.5 & 72.4 & 77.5 \\
\hline HIPC Initiative resources & 15.6 & 13.8 & 13.6 & 13.6 & 12.4 & 12.3 & 10.5 & 10.5 \\
\hline Externally financed & 135.2 & 149.1 & 142.2 & 136.8 & 213.3 & 170.0 & 196.9 & 209.7 \\
\hline Of which: grants & 95.7 & 119.0 & 107.2 & 106.3 & 143.3 & 120.0 & 128.6 & 137.0 \\
\hline Net lending & 0.0 & 0.0 & 0.0 & 0.0 & 0.0 & 0.0 & 0.0 & 0.0 \\
\hline Overall balance (commitment) & -129.4 & -167.0 & -157.1 & -105.9 & -346.9 & -318.5 & -258.5 & -260.7 \\
\hline Basic balance & 5.8 & -17.9 & -14.9 & 30.9 & -133.6 & -148.5 & -61.6 & -51.0 \\
\hline Change in payments arrears and float & -14.0 & -8.4 & -15.2 & -15.7 & -18.0 & -18.0 & -15.0 & -15.0 \\
\hline Of which: Domestic arrears & -14.0 & -14.8 & -15.2 & -15.7 & -18.0 & -17.8 & -15.0 & -15.0 \\
\hline Errors and omissions & 0.0 & 0.0 & 0.0 & 0.0 & 0.0 & 0.0 & 0.0 & 0.0 \\
\hline Overall balance (cash) & -143.8 & -175.4 & -172.3 & -121.8 & -364.9 & -336.3 & -273.5 & -275.7 \\
\hline Financing & 143.8 & 175.4 & 172.3 & 121.8 & 364.9 & 336.3 & 273.5 & 275.7 \\
\hline External financing & 235.0 & 197.1 & 176.3 & 167.6 & 304.2 & 256.8 & 261.6 & 272.9 \\
\hline Grants & 898.6 & 146.5 & 146.2 & 141.9 & 233.0 & 206.3 & 176.1 & 185.2 \\
\hline Budget financing & 18.6 & 27.5 & 38.9 & 35.6 & 89.7 & 86.3 & 47.5 & 48.2 \\
\hline Project financing & 95.7 & 119.0 & 107.2 & 106.3 & 143.3 & 120.0 & 128.6 & 137.0 \\
\hline HIPC Initiative assistance & $\ldots$ & $\ldots$ & $\ldots$ & $\ldots$ & $\ldots$ & $\ldots$ & $\ldots$ & $\ldots$ \\
\hline MDRI assistance ${ }^{1}$ & 784.3 & 0.0 & 0.0 & 0.0 & 0.0 & 0.0 & 0.0 & 0.0 \\
\hline Loans & 59.3 & 53.1 & 35.0 & 30.5 & 76.0 & 55.3 & 89.8 & 93.4 \\
\hline Of which: Budget financing & 19.8 & 23.0 & 0.0 & 0.0 & 6.0 & 5.3 & 21.5 & 20.7 \\
\hline Amortization & -726.0 & -6.1 & -8.2 & -8.8 & -8.7 & -8.5 & -8.7 & -10.1 \\
\hline Debt relief (incl. debt under discussion) & 3.1 & 3.5 & 3.4 & 4.0 & 3.9 & 3.7 & 4.4 & 4.4 \\
\hline Domestic financing & -91.2 & -21.7 & -4.0 & -45.9 & 60.7 & 79.5 & 11.9 & 2.8 \\
\hline Banking sector & -82.8 & -31.6 & -5.6 & -56.4 & 60.7 & 85.8 & 11.9 & 2.8 \\
\hline Of which: IMF & -61.4 & 5.8 & 5.3 & 5.3 & 4.8 & 5.0 & 4.9 & 0.3 \\
\hline Nonbanking sector & -8.4 & 9.9 & 1.6 & 10.5 & 0.0 & -6.3 & 0.0 & 0.0 \\
\hline
\end{tabular}

Sources: Nigerien authorities; and staff estimates.

${ }^{1}$ Multilateral Debt Relief Initiative stock estimates including cancellation of debt treated under the HIPC Initiative, shown on accrual basis. 
Table 2b. Niger: Financial Operations of the Central Government, 2006-11

\begin{tabular}{|c|c|c|c|c|c|c|c|c|}
\hline & \multirow{2}{*}{$\frac{2006}{\text { Est }}$} & \multirow{2}{*}{$\begin{array}{l}2007 \\
\text { Est. }\end{array}$} & \multicolumn{2}{|c|}{2008} & \multicolumn{2}{|c|}{2009} & \multirow{2}{*}{\multicolumn{2}{|c|}{$\begin{array}{ll}2010 & 2011 \\
\text { Projections } & \end{array}$}} \\
\hline & & & 1st Rev. & Est. & 1st Rev. & Proj. & & \\
\hline & \multicolumn{8}{|c|}{ (Percent of GDP) } \\
\hline Total revenue & 13.0 & 15.2 & 17.5 & 18.4 & 12.2 & 12.2 & 12.2 & 12.4 \\
\hline Tax revenue & 10.7 & 11.5 & 11.0 & 11.7 & 11.5 & 11.7 & 11.6 & 11.9 \\
\hline Of which: International trade & 5.0 & 4.9 & 4.3 & 4.6 & 4.2 & 4.2 & 4.3 & 4.3 \\
\hline Nontax revenue & 2.0 & 3.5 & 6.4 & 6.6 & 0.6 & 0.4 & 0.4 & 0.4 \\
\hline Of which: exceptional uranium receipts & 1.6 & 1.4 & 5.3 & 5.1 & 0.0 & 0.0 & 0.0 & 0.0 \\
\hline Special accounts revenue & 0.3 & 0.2 & 0.1 & 0.1 & 0.1 & 0.1 & 0.1 & 0.1 \\
\hline Settlement of reciprocal debts & 0.0 & 0.0 & 0.0 & 0.0 & 0.0 & 0.0 & 0.0 & 0.0 \\
\hline Total expenditure and net lending & 19.8 & 23.4 & 24.2 & 22.9 & 26.0 & 24.6 & 21.6 & 21.3 \\
\hline Of which: domestically financed & 12.7 & 16.1 & 18.1 & 17.1 & 17.5 & 18.0 & 14.4 & 14.2 \\
\hline Total current expenditure & 9.1 & 11.8 & 12.9 & 12.5 & 12.7 & 12.3 & 11.4 & 11.1 \\
\hline Budgetary expenditure & 8.6 & 9.8 & 11.9 & 11.0 & 11.7 & 11.1 & 10.9 & 10.6 \\
\hline Wages and salaries & 3.6 & 3.5 & 3.7 & 3.5 & 3.7 & 3.6 & 3.6 & 3.6 \\
\hline Materials and supplies & 2.5 & 3.0 & 3.1 & 2.6 & 3.5 & 3.4 & 3.3 & 3.1 \\
\hline Subsidies and transfers & 2.3 & 2.9 & 4.5 & 4.3 & 4.2 & 3.8 & 3.7 & 3.5 \\
\hline Of which: Transfers to other levels of gov $€$ & $\ldots$ & $\ldots$ & 0.3 & 0.3 & 0.0 & 0.0 & 0.0 & 0.0 \\
\hline Interest, scheduled & 0.3 & 0.3 & 0.3 & 0.2 & 0.3 & 0.2 & 0.3 & 0.3 \\
\hline Of which: External debt & 0.2 & 0.2 & 0.2 & 0.2 & 0.2 & 0.2 & 0.2 & 0.2 \\
\hline Adjustments and fiscal expenditures & 0.0 & 0.0 & 0.3 & 0.4 & 0.0 & 0.0 & 0.0 & 0.0 \\
\hline Special accounts expenditure & 0.5 & 2.0 & 1.0 & 1.5 & 1.0 & 1.2 & 0.5 & 0.6 \\
\hline Capital expenditure and net lending & 10.6 & 11.6 & 11.3 & 10.3 & 13.3 & 12.3 & 10.2 & 10.2 \\
\hline Capital expenditure & 10.6 & 11.6 & 11.3 & 10.3 & 13.3 & 12.3 & 10.2 & 10.2 \\
\hline Domestically financed & 2.7 & 3.6 & 4.6 & 4.0 & 4.3 & 5.2 & 2.6 & 2.6 \\
\hline \multicolumn{9}{|l|}{ Of which: Priority Investment Fund } \\
\hline HIPC Initiative resources & 0.8 & 0.7 & 0.6 & 0.6 & 0.5 & 0.5 & 0.4 & 0.4 \\
\hline Externally financed & 7.1 & 7.3 & 6.1 & 5.7 & 8.5 & 6.6 & 7.2 & 7.2 \\
\hline Of which: grants & 5.0 & 5.8 & 4.6 & 4.4 & 5.7 & 4.7 & 4.7 & 4.7 \\
\hline Net lending & 0.0 & 0.0 & 0.0 & 0.0 & 0.0 & 0.0 & 0.0 & 0.0 \\
\hline Budget reserve & 0.0 & 0.0 & $\ldots$ & $\ldots$ & $\ldots$ & $\ldots$ & $\ldots$ & $\ldots$ \\
\hline Overall balance (commitment) & -6.8 & -8.2 & -6.7 & -4.4 & -13.8 & -12.4 & -9.4 & -8.9 \\
\hline Basic balance & 0.3 & -0.9 & -0.6 & 1.3 & -5.3 & -5.8 & -2.2 & -1.7 \\
\hline Change in payments arrears and float & -0.7 & -0.4 & -0.7 & -0.7 & -0.7 & -0.7 & -0.5 & -0.5 \\
\hline Of which: Domestic arrears & -0.7 & -0.7 & -0.7 & -0.7 & -0.7 & -0.7 & -0.5 & -0.5 \\
\hline Errors and omissions & 0.0 & 0.0 & 0.0 & 0.0 & 0.0 & 0.0 & 0.0 & 0.0 \\
\hline Overall balance (cash) & -7.5 & -8.6 & -7.4 & -5.1 & -14.6 & -13.1 & -10.0 & -9.4 \\
\hline Financing & 7.5 & 8.6 & 7.4 & 5.1 & 14.6 & 13.1 & 10.0 & 9.4 \\
\hline Privatisations/sales of assets & $\ldots$ & 0.0 & 0.0 & 0.0 & 0.0 & 0.0 & 0.0 & 0.0 \\
\hline External financing & 12.3 & 9.7 & 7.6 & 7.0 & 12.1 & 10.0 & 9.5 & 9.3 \\
\hline Grants & 47.1 & 7.2 & 6.3 & 5.9 & 9.3 & 8.0 & 6.4 & 6.3 \\
\hline Budget financing & 1.0 & 1.4 & 1.7 & 1.5 & 3.6 & 3.4 & 1.7 & 1.6 \\
\hline Project financing & 5.0 & 5.8 & 4.6 & 4.4 & 5.7 & 4.7 & 4.7 & 4.7 \\
\hline HIPC Initiative assistance & $\ldots$ & $\ldots$ & $\ldots$ & $\ldots$ & $\ldots$ & $\ldots$ & $\ldots$ & $\ldots$ \\
\hline MDRI assistance ${ }^{1}$ & 41.1 & 0.0 & 0.0 & 0.0 & 0.0 & 0.0 & 0.0 & 0.0 \\
\hline Loans & 3.1 & 2.6 & 1.5 & 1.3 & 3.0 & 2.2 & 3.3 & 3.2 \\
\hline Of which: Budget financing & 1.0 & 1.1 & 0.0 & 0.0 & 0.2 & 0.2 & 0.8 & 0.7 \\
\hline Amortization & -38.1 & -0.3 & -0.4 & -0.4 & -0.3 & -0.3 & -0.3 & -0.3 \\
\hline Debt relief (incl. debt under discussion) & 0.2 & 0.2 & 0.1 & 0.2 & 0.2 & 0.1 & 0.2 & 0.1 \\
\hline Domestic financing & -4.8 & -1.1 & -0.2 & -1.9 & 2.4 & 3.1 & 0.4 & 0.1 \\
\hline Banking sector & -4.3 & -1.6 & -0.2 & -2.4 & 2.4 & 3.3 & 0.4 & 0.1 \\
\hline Of which: IMF & -3.2 & 0.3 & 0.2 & 0.2 & 0.2 & 0.3 & 0.2 & 0.0 \\
\hline Nonbanking sector & -0.4 & 0.5 & 0.1 & 0.4 & 0.0 & -0.2 & 0.0 & 0.0 \\
\hline
\end{tabular}

Sources: Nigerien authorities; and IMF staff estimates and projections.

1 Multilateral Debt Relief Initiative stock-of-debt operation, including cancellation of debt treated under the HIPC Initiative, shown on accrual basis. 
Table 2c. Niger: Financial Operations of the Central Government, Quarterly Projections for 2009

\begin{tabular}{|c|c|c|c|c|c|}
\hline & $\begin{array}{r}\text { Dec.08 } \\
\text { Actual }\end{array}$ & $\begin{array}{r}\text { Mar.09 } \\
\text { Proj. }\end{array}$ & $\begin{array}{r}\text { Jun.09 } \\
\text { Prog. }\end{array}$ & $\begin{array}{r}\text { Sep.09 } \\
\text { Prog. }\end{array}$ & $\begin{array}{r}\text { Dec.09 } \\
\text { Prog. }\end{array}$ \\
\hline Total revenue & 442.0 & 81.6 & 147.1 & 230.3 & 312.3 \\
\hline Tax revenue & 281.1 & 78.3 & 141.6 & 221.1 & 299.3 \\
\hline Of which : International trade & 110.7 & 26.9 & 49.8 & 78.6 & 107.5 \\
\hline Nontax revenue & 158.8 & 2.5 & 4.1 & 7.1 & 10.1 \\
\hline Special accounts revenue & 2.1 & 0.7 & 1.5 & 2.2 & 2.9 \\
\hline Total expenditure and net lending & 547.9 & 157.7 & 315.4 & 473.1 & 630.8 \\
\hline Total current expenditure & 300.8 & 79.0 & 158.0 & 237.0 & 316.0 \\
\hline Budgetary expenditure & 256.6 & 71.4 & 142.8 & 214.2 & 285.6 \\
\hline Wages and salaries & 83.8 & 23.3 & 46.7 & 70.0 & 93.3 \\
\hline Materials and supplies & 63.4 & 21.9 & 43.8 & 65.7 & 87.6 \\
\hline Subsidies and transfers & 94.6 & 24.6 & 49.2 & 73.8 & 98.4 \\
\hline Interest, scheduled & 5.5 & 1.6 & 3.1 & 4.7 & 6.3 \\
\hline External debt & 3.7 & 1.1 & 2.3 & 3.4 & 4.5 \\
\hline Adjustments & 9.3 & 0.0 & 0.0 & 0.0 & 0.0 \\
\hline Special accounts expenditure & 36.7 & 7.6 & 15.2 & 22.8 & 30.4 \\
\hline Capital expenditure and net lending & 247.1 & 78.7 & 157.4 & 236.1 & 314.8 \\
\hline Capital expenditure & 247.1 & 78.7 & 157.4 & 236.1 & 314.8 \\
\hline Domestically financed & 96.7 & 33.1 & 66.3 & 99.4 & 132.5 \\
\hline Grants & 106.3 & 30.0 & 60.0 & 90.0 & 120.0 \\
\hline Loans & 30.5 & 12.5 & 25.0 & 37.5 & 50.0 \\
\hline HIPCInitiative resources & 13.6 & 3.1 & 6.2 & 9.2 & 12.3 \\
\hline Overall balance (commitment) & -105.9 & -76.1 & -168.3 & -242.8 & -318.5 \\
\hline Basic balance & 30.9 & -33.6 & -83.3 & -115.3 & -148.5 \\
\hline Change in payments arrears & -15.9 & -2.0 & -6.0 & -8.0 & -17.8 \\
\hline Domestic arrears & -15.7 & -2.0 & -6.0 & -8.0 & -17.8 \\
\hline Overall balance (cash) & -121.8 & -78.1 & -174.3 & -250.8 & -336.3 \\
\hline Financing & 121.8 & 78.1 & 174.3 & 250.8 & 336.3 \\
\hline External financing & 167.6 & 65.8 & 117.0 & 164.9 & 256.8 \\
\hline Grants & 141.9 & 54.5 & 94.4 & 131.0 & 206.3 \\
\hline Budget financing & 35.6 & 24.5 & 34.4 & 41.0 & 86.3 \\
\hline Project financing & 106.3 & 30.0 & 60.0 & 90.0 & 120.0 \\
\hline HIPC Initiative assistance & 0.0 & 0.0 & 0.0 & 0.0 & 0.0 \\
\hline IMF MDRI assistance & 0.0 & 0.0 & 0.0 & 0.0 & 0.0 \\
\hline Loans & 30.5 & 12.5 & 25.0 & 37.5 & 55.3 \\
\hline Budget financing & 0.0 & 0.0 & 0.0 & 0.0 & 5.3 \\
\hline Project financing & 30.5 & 12.5 & 25.0 & 37.5 & 50.0 \\
\hline Amortization & -8.8 & -2.1 & -4.3 & -6.4 & -8.5 \\
\hline Debt relief & 4.0 & 0.9 & 1.9 & 2.8 & 3.7 \\
\hline Domestic financing & -45.9 & 12.3 & 57.3 & 85.9 & 79.5 \\
\hline Banking sector & -56.4 & 12.3 & 57.3 & 85.9 & 85.8 \\
\hline Of which: IMF & 5.3 & 0.0 & 2.5 & 2.5 & 5.0 \\
\hline Non banking sector & 10.5 & 0.0 & 0.0 & 0.0 & -6.3 \\
\hline
\end{tabular}

Sources: Nigerien authorities; and staff estimates. 
Table 3. Niger: Monetary Survey, 2006-11

\begin{tabular}{|c|c|c|c|c|c|c|c|c|}
\hline & \multirow[t]{2}{*}{2006} & \multirow[t]{2}{*}{2007} & \multicolumn{2}{|c|}{2008} & \multicolumn{2}{|c|}{2009} & \multirow{2}{*}{$\begin{array}{c}2010 \\
\text { Projections }\end{array}$} & \multirow[t]{2}{*}{2011} \\
\hline & & & 1st Rev. & Est. & 1st Rev. & Projections & & \\
\hline & \multicolumn{8}{|c|}{ (CFAF billions; end-of-period) } \\
\hline Net foreign assets & 162.5 & 231.8 & 301.3 & 287.3 & 284.1 & 270.3 & 286.8 & 305.9 \\
\hline BCEAO & 168.4 & 240.7 & 337.3 & 299.6 & 322.0 & 290.6 & 309.1 & 330.1 \\
\hline Commercial banks & -5.8 & -9.0 & -36.0 & -12.3 & -38.0 & -20.3 & -22.3 & -24.3 \\
\hline Net domestic assets & 126.5 & 123.9 & 140.0 & 113.6 & 239.9 & 225.9 & 285.6 & 345.5 \\
\hline Domestic credit & 151.7 & 141.6 & 157.7 & 147.9 & 257.5 & 270.2 & 332.9 & 392.8 \\
\hline Net bank claims on government & -7.9 & -50.4 & -56.0 & -114.7 & 4.8 & -29.2 & -17.3 & -14.5 \\
\hline BCEAO & 0.6 & -31.2 & -34.7 & -98.3 & 20.9 & -13.5 & -2.2 & 0.2 \\
\hline Of which: statutory advances & 35.2 & 33.7 & 30.5 & 33.3 & 27.3 & 30.0 & 26.8 & 23.6 \\
\hline IMF resources & 13.8 & 19.6 & 24.9 & 24.8 & 29.7 & 32.2 & 37.1 & 37.4 \\
\hline Commercial banks & -10.4 & -20.6 & -23.2 & -20.3 & -18.4 & -20.3 & -20.3 & -20.3 \\
\hline Other & 1.8 & 1.4 & 1.9 & 3.9 & 2.3 & 4.6 & 5.2 & 5.7 \\
\hline Credit to the economy & 159.6 & 191.9 & 213.7 & 262.6 & 252.8 & 299.4 & 350.2 & 407.3 \\
\hline Other items, net & -25.2 & -17.6 & -17.6 & -34.3 & -17.6 & -44.3 & -47.3 & -47.3 \\
\hline Money and quasi-money & 289.1 & 355.7 & 441.3 & 401.0 & 523.9 & 496.2 & 572.4 & 651.4 \\
\hline Currency outside banks & 132.9 & 132.8 & 156.1 & 147.1 & 175.7 & 169.4 & 191.4 & 216.3 \\
\hline \multicolumn{9}{|l|}{ Private deposits with ONPE } \\
\hline (Postal savings institution) & 1.8 & 1.4 & 1.9 & 3.9 & 2.3 & 4.6 & 5.2 & 5.7 \\
\hline Deposits with banks & 154.4 & 221.5 & 283.3 & 250.0 & 346.0 & 322.2 & 375.8 & 429.4 \\
\hline & \multicolumn{8}{|c|}{ (Annual change, in percent of beginning-of-period broad money, unless otherwise indicated) } \\
\hline Net foreign assets & 33.6 & 23.9 & 19.6 & 15.6 & -3.9 & -4.2 & 3.3 & 3.3 \\
\hline BCEAO & 40.7 & 25.0 & 27.1 & 16.6 & -3.5 & -2.2 & 3.7 & 3.7 \\
\hline Commercial banks & -7.1 & -1.1 & -7.6 & -0.9 & -0.5 & -2.0 & -0.4 & -0.3 \\
\hline Net domestic assets & -17.3 & -0.9 & 4.5 & -2.9 & 22.6 & 28.0 & 12.0 & 10.5 \\
\hline Domestic credit & -16.1 & -3.5 & 4.5 & 1.8 & 22.6 & 30.5 & 12.6 & 10.5 \\
\hline Net bank claims on the government & -31.6 & -14.7 & -1.6 & -18.1 & 13.8 & 21.3 & 2.4 & 0.5 \\
\hline BCEAO & -30.0 & -11.0 & -1.0 & -18.9 & 12.6 & 21.1 & 2.3 & 0.4 \\
\hline Of which: statutory advances & 1.3 & -0.5 & -0.9 & -0.1 & -0.7 & -0.8 & -0.6 & -0.6 \\
\hline Commercial banks & -1.3 & -3.5 & -0.8 & 0.1 & 1.1 & 0.0 & 0.0 & 0.0 \\
\hline Other & -0.3 & -0.1 & 0.2 & 0.7 & 0.1 & 0.2 & 0.1 & 0.1 \\
\hline Credit to the economy & 15.4 & 11.2 & 6.1 & 19.9 & 8.9 & 9.2 & 10.2 & 10.0 \\
\hline Other items, net & -1.2 & 2.6 & 0.0 & -4.7 & 0.0 & -2.5 & -0.6 & 0.0 \\
\hline Broad money & 16.2 & 23.0 & 24.1 & 12.7 & 18.7 & 23.8 & 15.4 & 13.8 \\
\hline \multicolumn{9}{|l|}{ Memorandum items: } \\
\hline \multicolumn{9}{|l|}{ Velocity of broad money } \\
\hline (in percent) & 6.6 & 5.7 & 5.3 & 6.0 & 4.8 & 5.2 & 4.8 & 4.5 \\
\hline \multicolumn{9}{|l|}{ Credit to the economy } \\
\hline (Change, in percent) & 31.7 & 20.2 & 11.3 & 36.8 & 18.3 & 14.0 & 17.0 & 16.3 \\
\hline
\end{tabular}

Sources: BCEAO; and IMF staff estimates and projections. 
Table 4. Niger: Balance of Payments, 2006-11

\begin{tabular}{|c|c|c|c|c|c|c|c|c|}
\hline & \multirow[t]{2}{*}{2006} & \multirow[t]{2}{*}{2007} & \multicolumn{2}{|c|}{2008} & \multicolumn{2}{|l|}{2009} & 2010 & 2011 \\
\hline & & & 1st Rev. & $\overline{\text { Est. }}$ & 1st Rev. & Proj. & \multicolumn{2}{|c|}{ Projections } \\
\hline & \multicolumn{8}{|c|}{ (Billions of CFA francs, unless otherwise indicated) } \\
\hline Current account balance & -185.5 & -183.4 & -230.2 & -301.1 & -564.8 & -542.6 & -831.8 & -937.6 \\
\hline Balance on goods and services & -249.1 & -247.8 & -330.0 & -372.4 & -726.8 & -670.9 & -924.2 & -1034.0 \\
\hline Balance on goods & -125.7 & -111.1 & -147.7 & -174.2 & -448.4 & -358.7 & -495.5 & -565.9 \\
\hline Exports, f.o.b & 265.6 & 318.3 & 393.1 & 397.9 & 418.5 & 390.1 & 450.3 & 463.6 \\
\hline Uranium & 79.6 & 143.1 & 178.2 & 201.0 & 192.2 & 188.4 & 229.0 & 229.0 \\
\hline Cattle & 35.5 & 37.8 & 37.8 & 42.4 & 39.1 & 44.1 & 47.1 & 50.5 \\
\hline Cowpeas & 18.5 & 19.2 & 23.2 & 36.7 & 25.0 & 33.5 & 29.4 & 31.5 \\
\hline Onions & 37.7 & 42.1 & 41.3 & 42.9 & 44.9 & 46.6 & 49.9 & 53.5 \\
\hline Gold & 24.3 & 25.5 & 26.0 & 26.3 & 25.2 & 27.9 & 41.8 & 42.3 \\
\hline \multicolumn{9}{|l|}{ Oil } \\
\hline Other exports & 70.0 & 50.5 & 86.6 & 48.6 & 92.1 & 49.6 & 53.0 & 56.8 \\
\hline Imports, f.o.b & 391.3 & 429.4 & 540.8 & 572.1 & 866.8 & 748.8 & 945.8 & 1029.5 \\
\hline Food products & 97.7 & 86.5 & 94.7 & 121.5 & 85.2 & 111.8 & 115.0 & 120.7 \\
\hline Petroleum products & 48.4 & 64.8 & 82.7 & 94.0 & 90.0 & 62.5 & 83.7 & 94.9 \\
\hline Intermediate goods & 21.4 & 27.5 & 31.0 & 34.3 & 35.7 & 84.3 & 121.5 & 133.7 \\
\hline Capital goods & 151.1 & 182.2 & 193.8 & 248.9 & 503.4 & 393.6 & 514.0 & 560.3 \\
\hline Other products & 72.7 & 68.3 & 138.5 & 73.3 & 152.5 & 96.7 & 111.6 & 119.8 \\
\hline Services and income (net) & -123.4 & -136.7 & -182.2 & -198.3 & -278.5 & -312.1 & -428.7 & -468.1 \\
\hline Services (net) & -124.1 & -136.5 & -172.6 & -190.8 & -269.5 & -269.2 & -358.1 & -392.1 \\
\hline Income (net) & 0.6 & -0.2 & -9.7 & -7.4 & -9.0 & -42.9 & -70.6 & -75.9 \\
\hline Of which: interest on external public debt & -3.7 & -4.4 & -4.5 & -3.7 & -4.9 & -4.5 & -6.3 & -7.2 \\
\hline Unrequited current transfers (net) & 63.6 & 64.5 & 99.8 & 71.3 & 162.0 & 128.3 & 92.4 & 96.4 \\
\hline Private (net) & 41.6 & 44.0 & 43.5 & 47.0 & 52.4 & 55.4 & 59.2 & 63.4 \\
\hline Public (net) & 22.1 & 20.5 & 56.3 & 24.3 & 109.6 & 73.0 & 33.2 & 32.9 \\
\hline Of which: grants for budgetary assistance & 18.6 & 27.5 & 38.9 & 35.6 & 89.7 & 86.3 & 47.5 & 48.2 \\
\hline Capital and financial account & 293.1 & 255.8 & 326.7 & 356.6 & 549.6 & 533.4 & 850.3 & 958.6 \\
\hline Capital account & 912.0 & 153.4 & 114.5 & 113.4 & 151.7 & 128.4 & 137.6 & 146.6 \\
\hline Private capital transfers & 7.1 & 6.9 & 7.3 & 7.1 & 8.4 & 8.4 & 8.9 & 9.6 \\
\hline Project grants & 95.7 & 119.0 & 107.2 & 106.3 & 143.3 & 120.0 & 128.6 & 137.0 \\
\hline Nonproduced, nonfinancial assets & 30.4 & 27.5 & 0.0 & 0.0 & 0.0 & 0.0 & 0.0 & 0.0 \\
\hline Debt cancellation ${ }^{1}$ & 778.8 & 0.0 & 0.0 & 0.0 & 0.0 & 0.0 & 0.0 & 0.0 \\
\hline Financial account & -618.9 & 102.4 & 212.2 & 243.2 & 397.9 & 405.0 & 712.7 & 812.0 \\
\hline Direct investment & 26.9 & 58.0 & 188.3 & 225.0 & 333.6 & 348.9 & 635.3 & 714.3 \\
\hline Portfolio investment & -2.0 & -3.0 & 1.8 & -3.2 & 2.0 & 2.0 & 2.0 & 2.0 \\
\hline Other investment & -643.8 & 47.4 & 22.1 & 21.4 & 62.3 & 54.1 & 75.5 & 95.8 \\
\hline Public sector (net) & -666.7 & 47.1 & 26.8 & 21.7 & 67.3 & 46.8 & 81.1 & 83.3 \\
\hline Disbursements & 59.3 & 53.1 & 35.0 & 30.5 & 76.0 & 55.3 & 89.8 & 93.4 \\
\hline Loans for budgetary assistance & 19.8 & 23.0 & 0.0 & 0.0 & 6.0 & 5.3 & 21.5 & 20.7 \\
\hline Project loans & 39.5 & 30.1 & 35.0 & 30.5 & 70.0 & 50.0 & 68.3 & 72.7 \\
\hline Amortization & 726.0 & 6.1 & 8.2 & 8.8 & 8.7 & 8.5 & 8.7 & 10.1 \\
\hline Other (net) & 22.9 & 0.4 & -4.7 & -0.3 & -5.0 & 7.3 & -5.6 & 12.5 \\
\hline Errors and omissions & -9.4 & -3.6 & 0.0 & 0.0 & 0.0 & 0.0 & 0.0 & 0.0 \\
\hline Overall balance & 98.2 & 68.9 & 96.5 & 55.5 & -15.2 & -9.2 & 18.5 & 21.0 \\
\hline Financing & -98.2 & -68.9 & -96.5 & -55.5 & 15.2 & 9.0 & -18.5 & -21.0 \\
\hline $\begin{array}{l}\text { Net foreign assets (BCEAO) } \\
\text { Assets }\end{array}$ & -101.2 & -72.4 & -96.5 & -58.9 & 15.2 & 9.0 & -18.5 & -21.0 \\
\hline \multicolumn{9}{|l|}{ Liabilities } \\
\hline Of which: net use of Fund resources & -56.4 & 5.7 & 5.3 & 5.3 & 4.8 & 5.0 & 4.9 & 0.3 \\
\hline Purchases & 4.5 & 5.7 & 5.3 & 5.3 & 4.8 & 5.0 & 4.9 & 2.5 \\
\hline Repurchases & -60.9 & 0.0 & 0.0 & 0.0 & 0.0 & 0.0 & -0.1 & -2.2 \\
\hline Rescheduling obtained & 3.0 & 3.5 & 0.0 & 3.4 & 0.0 & 0.0 & 0.0 & 0.0 \\
\hline Change in arrears & 0.0 & 0.0 & 0.0 & 0.0 & 0.0 & 0.0 & 0.0 & 0.0 \\
\hline & \multicolumn{8}{|c|}{ (Percent of GDP, unless otherwise indicated) } \\
\hline \multicolumn{9}{|l|}{ Memorandum items: } \\
\hline \multicolumn{9}{|l|}{ External current account balance } \\
\hline Including official grants (percent of GDP) & -9.7 & -9.0 & -9.9 & -12.6 & -22.5 & -21.2 & -30.3 & -32.0 \\
\hline Excluding official grants (percent of GDP) & -10.9 & -10.0 & -12.3 & -13.6 & -26.9 & -24.0 & -31.5 & -33.2 \\
\hline Net Foreign Assets of BCEAO (months of imports) & 3.6 & 4.8 & 5.3 & 4.4 & 3.2 & 3.3 & 2.7 & 2.7 \\
\hline GDP (in CFAF billions) & 1906.4 & 2034.8 & 2333.1 & 2397.7 & 2507.6 & 2564.0 & 2748.3 & 2926.7 \\
\hline Petroleum price (US $\$$ per barrel) & 64.3 & 71.1 & 99.8 & 97.0 & 68.0 & 44.0 & 52.0 & 57.5 \\
\hline Uranium price (000's FCFA/kg.) & 25.2 & 41.9 & 61.9 & 63.2 & 58.1 & 56.9 & 59.0 & 59.0 \\
\hline Uranium price (US $\$ / \mathrm{lb}$ ) & 18.6 & 33.7 & & 54.5 & & & $\ldots$ & \\
\hline NPV of external debt (percent of exports) & 62.6 & 60.4 & 47.9 & 47.9 & 57.4 & 57.4 & 59.6 & 65.2 \\
\hline Exchange rate (CFAF per US $\$$, annual average) & 522.4 & 478.6 & $\ldots$ & 445.7 & $\ldots$ & $\ldots$ & $\ldots$ & $\ldots$ \\
\hline
\end{tabular}

Sources: Nigerien authorities; and IMF staff estimates and projections.

${ }^{1}$ Multilateral Debt Relief Initiative stock-of-debt operation, including cancellation of debt treated under the enhanced HIPC initiative. 
Table 5 . Budget Expenditure on Social and Rural Sectors, 2005-08

(Millions of CFA francs)

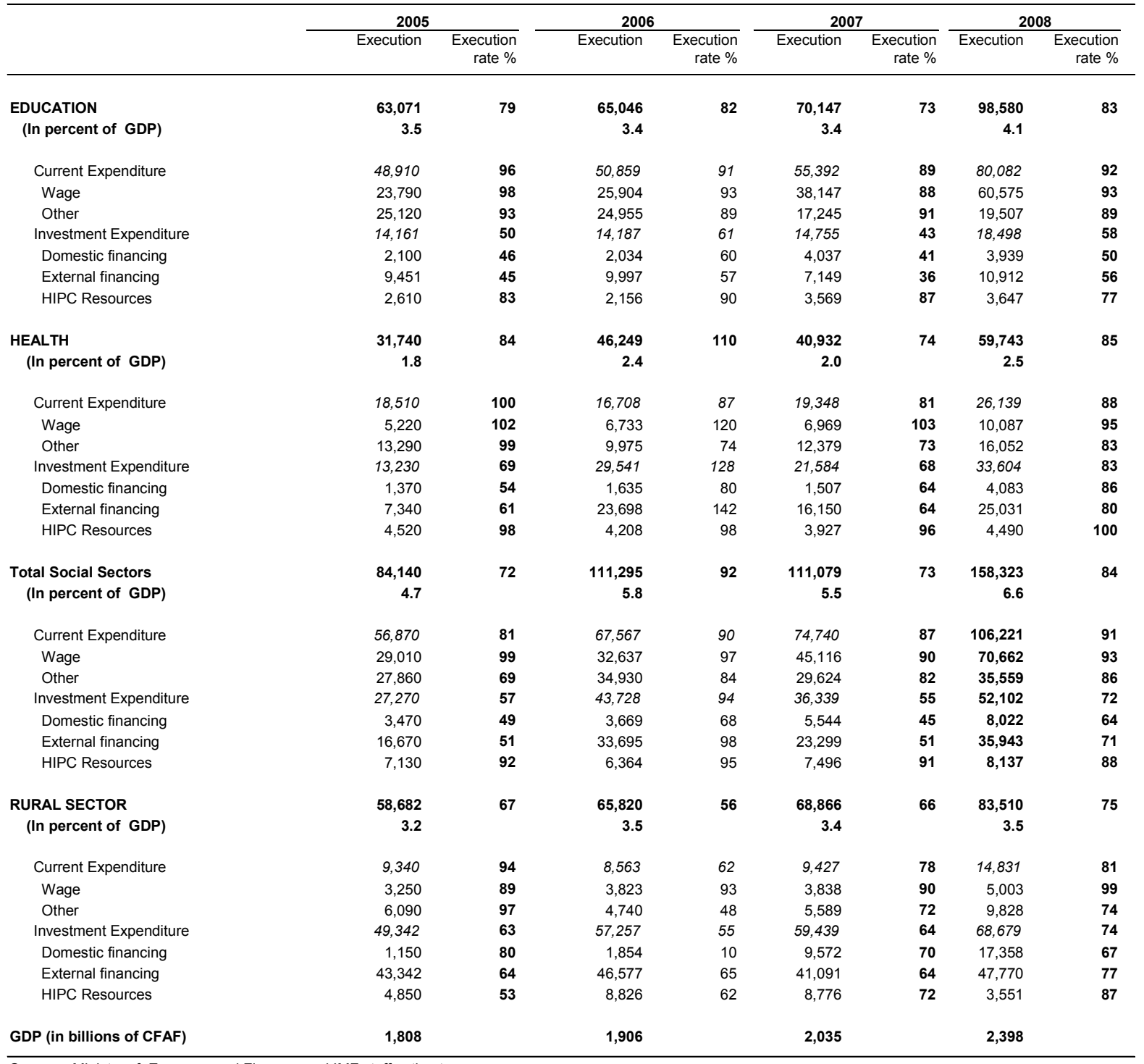

Sources: Ministry of Economy and Finance; and IMF staff estimates. 
Table 6. Niger: Millennium Development Goals, 1990-2007

\begin{tabular}{|c|c|c|c|c|}
\hline & 1990 & 1995 & 2000 & 2007 \\
\hline \multicolumn{5}{|l|}{ Goal 1: Eradicate extreme poverty and hunger } \\
\hline Employment to population ratio, $15+$, total $(\%)$ & 78 & 79 & 79 & 79 \\
\hline Employment to population ratio, ages $15-24$, total (\%) & 68 & 70 & 70 & 71 \\
\hline Income share held by lowest $20 \%$ & 7.5 & 2.6 & .. & .. \\
\hline Malnutrition prevalence, weight for age (\% of children under 5 ) & 41.0 & .. & 43.6 & 39.9 \\
\hline Poverty headcount ratio at national poverty line ( $\%$ of population) & .. & 63.0 & .. & .. \\
\hline Prevalence of undernourishment (\% of population) & 41 & 42 & .. & .. \\
\hline $\begin{array}{l}\text { Vulnerable employment, total (\% of total employment) } \\
\text { Goal 2: Achieve universal primary education }\end{array}$ & .. & .. & .. & .. \\
\hline Literacy rate, youth female (\% of females ages $15-24)$ & .. & .. & .. & 23 \\
\hline Literacy rate, youth male ( $\%$ of males ages $15-24)$ & .. & .. & .. & 52 \\
\hline Persistence to last grade of primary, total (\% of cohort) & .. & .. & 69 & 53 \\
\hline Primary completion rate, total ( $\%$ of relevant age group) & 15 & 13 & 18 & 43 \\
\hline $\begin{array}{l}\text { Total enrollment, primary (\% gross) } \\
\text { Goal 3: Promote gender equality and empower women }\end{array}$ & .. & .. & 37 & 62 \\
\hline Proportion of seats held by women in national parliament (\%) & 5 & .. & 1 & 12 \\
\hline Ratio of female to male enrollments in tertiary education & .. & .. & .. & 29 \\
\hline Ratio of female to male primary enrollment & 61 & .. & 69 & 73 \\
\hline Ratio of female to male secondary enrollment & 37 & .. & 61 & 63 \\
\hline Ratio of young literate females to males (\% ages $15-24)$ & .. & .. & .. & 44 \\
\hline $\begin{array}{l}\text { Share of women employed in the nonagricultural sector (\% of total nonagricultural employment) } \\
\text { Goal 4: Reduce child mortality }\end{array}$ & 11.0 & .. &.. & .. \\
\hline Immunization, measles ( $\%$ of children ages $12-23$ months) & 25 & 40 & 34 & 47 \\
\hline Mortality rate, infant (per 1,000 live births) & 191 & 176 & 159 & 81 \\
\hline Mortality rate, under-5 (per 1,000$)$ & 320 & 295 & 270 & 196 \\
\hline \multicolumn{5}{|l|}{ Goal 5: Improve maternal health } \\
\hline Adolescent fertility rate (births per 1,000 women ages $15-19$ ) & .. & 229 & 224 & 201 \\
\hline Births attended by skilled health staff ( $\%$ of total) & 15 & .. & 16 & 18 \\
\hline Contraceptive prevalence ( $\%$ of women ages $15-49$ ) & 4 & .. & 14 & 11 \\
\hline Maternal mortality ratio (modeled estimate, per 100,000 live births) & .. & .. &.. & 648 \\
\hline Pregnant women receiving prenatal care $(\%)$ & 30 & .. & 41 & 46 \\
\hline \multicolumn{5}{|l|}{ Goal 6: Combat HIVIAIDS, malaria, and other diseases } \\
\hline Children with fever receiving antimalarial drugs ( $\%$ of children under age 5 with fever) & .. & .. & 48 & 33 \\
\hline Condom use, population ages $15-24$, female ( $\%$ of females ages $15-24)$ & .. & .. & .. & .. \\
\hline Condom use, population ages $15-24$, male $(\%$ of males ages $15-24)$ & .. & .. & .. & .. \\
\hline Incidence of tuberculosis (per 100,000 people) & 124 & 137 & 153 & 174 \\
\hline Prevalence of HIV, female (\% ages $15-24)$ & .. & .. &.. & 0.5 \\
\hline Prevalence of HIV, total (\% of population ages $15-49$ ) & .. & .. & 0.7 & 0.8 \\
\hline \multicolumn{4}{|l|}{ Goal 7: Ensure environmental sustainability } & 49 \\
\hline Annual freshwater withdrawals, total ( $\%$ of internal resources) & .. & .. & 62.3 & .. \\
\hline CO2 emissions (kg per PPP \$ of GDP) & 0.3 & 0.3 & 0.2 & .. \\
\hline CO2 emissions (metric tons per capita) & 0.1 & 0.1 & 0.1 & .. \\
\hline Forest area ( $\%$ of land area) & 2 & .. & 1 & 1 \\
\hline Improved sanitation facilities ( $\%$ of population with access) & 3 & 5 & 5 & 7 \\
\hline Improved water source (\% of population with access) & .. & .. &.. & 68.7 \\
\hline Marine protected areas, ( $\%$ of surface area) & .. & .. &.. & .. \\
\hline $\begin{array}{l}\text { Nationally protected areas (\% of total land area) } \\
\text { Goal 8: Develop a global partnership for development }\end{array}$ & .. & .. & .. & .. \\
\hline Aid per capita (current US\$) & 50 & 29 & 19 & 29 \\
\hline Debt service (PPG and IMF only, \% of exports of G\&S, excl. workers' remittances) & 6.6 & 7.8 & 7.9 & 2.3 \\
\hline Internet users (per 100 people) & 0.0 & 0.0 & 0.0 & 0.3 \\
\hline Mobile phone subscribers (per 100 people) & 0.0 & 0.0 & 0.0 & 6.3 \\
\hline Telephone mainlines (per 100 people) & 0.1 & 0.1 & 0.2 & 0.2 \\
\hline \multicolumn{5}{|l|}{ Other } \\
\hline Fertility rate, total (births per woman) & 7.9 & 7.7 & 7.5 & 7.0 \\
\hline GNI per capita, Atlas method (current US\$) & 300 & 190 & 170 & 280 \\
\hline GNI, Atlas method (current US\$) (billions) & 2.4 & 1.8 & 1.9 & 4.0 \\
\hline Gross capital formation (\% of GDP) & 8.1 & 7.3 & 11.4 & 23.1 \\
\hline Life expectancy at birth, total (years) & 47 & 50 & 53 & 56 \\
\hline Literacy rate, adult total ( $\%$ of people ages 15 and above) & .. & .. & .. & 29 \\
\hline Population, total (millions) & 7.8 & 9.3 & 11.1 & 14.2 \\
\hline Trade ( $\%$ of GDP) & 37.0 & 41.5 & 43.5 & 40.1 \\
\hline
\end{tabular}

Sources: Nigerien authorites; and World Bank, World Development Indicators (2008).

${ }^{1}$ Figures in italics refer to periods other than those specified. 
Table 7. Niger: Key Social Indicators, 2006-08

$$
\text { (In percent) }
$$

\begin{tabular}{lcc}
\hline & 2006 & $2007-08$ \\
\hline Admission rate to first grade & 64.7 & 77.8 \\
Gross enrollment ratio in primary school & 57.1 & 62.6 \\
Primary completion rate & 42.5 & 42.7 \\
Gross enrollment rate in secondary school & 14.9 & 17.0 \\
Secondary completion rate & 8.1 & 11.3 \\
Gender gap in secondary enrollment & 58.4 & 55.1 \\
Rate of assisted birth & 14.0 & 20.0 \\
Utilization rate of health facilities & 18.0 & 30.0 \\
Prenatal consultation coverage & 36.0 & 86.0 \\
\hline Sourc: Nigerien
\end{tabular}

Source: Nigerien authorities, 2007 Annual Progress Report, PRSP. 
Table 8. Niger: Proposed Scheduled Disbursements Under the PRGF Arrangement, 2008-11

\begin{tabular}{|c|c|c|c|}
\hline $\begin{array}{l}\text { Amount } \\
\text { (Millions) }\end{array}$ & Date Available & $\begin{array}{l}\text { Conditions Necessary for } \\
\text { Disbursement }^{1}\end{array}$ & Disbursement Date \\
\hline SDR 3.29 & June 2, 2008 & $\begin{array}{l}\text { Executive Board approval of the } \\
\text { three year PRGF arrangement. }\end{array}$ & June 18, 2008 \\
\hline SDR 3.29 & September 30, 2008 & $\begin{array}{l}\text { Observance of the June } 30,2008 \text { and } \\
\text { continuous quantitative performance } \\
\text { criteria, the end-September structural } \\
\text { performance criterion, and completion } \\
\text { of the first review under the } \\
\text { arrangement. }\end{array}$ & January 2, 2009 \\
\hline SDR 3.29 & March 31, 2009 & $\begin{array}{l}\text { Observance of the December } 31,2008 \text {, } \\
\text { and continuous quantitative } \\
\text { performance criteria, the end-November } \\
2008 \text { structural performance criterion, } \\
\text { and completion of the second review } \\
\text { under the arrangement. }\end{array}$ & \\
\hline SDR 3.29 & September 30, 2009 & $\begin{array}{l}\text { Observance of the June } 30,2009 \text { and } \\
\text { continuous quantitative performance } \\
\text { criteria, and completion of the third } \\
\text { review under the arrangement. }\end{array}$ & \\
\hline SDR 3.29 & March 31, 2010 & $\begin{array}{l}\text { Observance of the December 31, } 2009 \\
\text { and continuous quantitative } \\
\text { performance criteria, the end-December } \\
2009 \text { structural performance criteria, } \\
\text { and completion of the fourth review } \\
\text { under the arrangement. }\end{array}$ & \\
\hline SDR 3.29 & September 30, 2010 & $\begin{array}{l}\text { Observance of the June } 30,2010 \text { and } \\
\text { continuous performance criteria, and } \\
\text { completion of the fifth review under the } \\
\text { arrangement. }\end{array}$ & \\
\hline SDR 3.29 & March 31, 2011 & $\begin{array}{l}\text { Observance of the December } 31,2010 \\
\text { and continuous performance criteria, } \\
\text { and completion of the sixth review } \\
\text { under the arrangement. }\end{array}$ & \\
\hline
\end{tabular}

Source: IMF.

${ }^{1}$ In addition to the generally-applicable conditions under the PRGF arrangement. 


\section{APPENDIX -LETTER OF INTENT}

Niamey, 27 April, 2009

Mr. Dominique Strauss-Kahn

Managing Director

International Monetary Fund

Washington, D.C. 20431

Dear Mr. Strauss-Kahn:

1. This letter provides an update to the letter of intent and the Memorandum of Economic Policies of December 3, 2008. The program supported by the Poverty Reduction and Growth Facility (PRGF) approved on May 28, 2008 was implemented satisfactorily during the second half of 2008 and the first months of 2009. All the performance criteria for end-2008 have been observed. The criterion on domestic financing of the government was met with a comfortable margin as a result of significant excess in revenue and lower-thanexpected expenditures. Two of the structural benchmarks up to March 2009 have been observed. The third, concerning the presentation of the 2009-2012 priority capital expenditure program as an annex to the 2009 Budget Law has not been observed, as the medium-term public expenditure framework is still currently under preparation.

\section{Macroeconomic and fiscal developments in $\mathbf{2 0 0 8}$}

2. GDP growth in 2008 exceeded program expectations as a result of an exceptional agricultural season characterized by good rainfall distribution over time and across the various regions of the country. Agricultural production rose by 25 percent, compared with 11.6 percent initially forecast, with record harvests of millet, sorghum, wheat, rice, corn, fonio, and cowpeas. GDP growth in real terms has therefore been provisionally estimated at 9.5 percent. The expected increase in public and private investments materialized (20.5 percent increase in real terms), driven by the launch of externally-financed road building projects and the coming on stream of private investments in oil drilling, refinery construction and the new Imouraren uranium mine, in addition to investments made by the existing uranium companies. The investment-to-GDP ratio is estimated at 26.5 percent in 2008 compared with 23.5 percent in 2007.

3. Regarding inflation, the consumer price index rose by 13.6 percent in the 12 -month to December 2008 and by 11.3 percent on average. At end-February 2009, the year-on-year figure dropped to 10.4 percent as the good harvest lowered food prices. This fall in cereal prices is, however, less substantial than expected and generally observed at this time of year. 
Retail prices of petroleum products fell sharply, by around 20 percent, between November 2008 and March 2009.

4. Bank credit to the economy grew at a sustained pace in 2008, up 37 percent, with the transport, trade, and telecommunications sectors accounting for the fastest growth. Bank deposits were up 13 percent over the year. Banks were able to secure medium-term external credit lines, and reduced their short term deposits with the central bank, which nevertheless ended the year at comfortable levels (13 percent of deposits). Banks sought no central bank liquidity injections in either 2008 or the early months of 2009 , but have made use of normal rediscounts.

5. In 2008, based on preliminary figures, the current account deficit of the balance of payments is estimated at 14 percent of GDP, compared to 9.9 percent projected in the program and 10 percent in 2007. This worsening of the deficit is attributable to sizable capital goods imports and the rise in oil and food prices. The build up of the BCEAO's external reserves attributable to Niger was lower than expected (CFAF 59 billion compared with 97 billion).

6. Fiscal performance for 2008 has been satisfactory. The basic fiscal deficit amounted to CFAF 31 billion (1.3 percent of GDP), significantly higher than the deficit projections of 0.6 percent of GDP. Tax revenue exceeded the target by CFAF 22 billion, reaching 11.7 percent of revised GDP, compared to 11 percent programmed. This good performance is attributable, in part, to an acceleration of imports between March and September to take advantage of tax breaks on basic foodstuffs (rice, milk, sugar, cooking oil) as well as to higher-than-expected revenue from corporate profit tax (ISB), domestic VAT, excise taxes, and stamp duty. Non-tax revenue also exceeded targets, buoyed by higher dividends and interest earnings on government deposits at the BCEAO. On the expenditure side, expenditures related to the purchase of goods and services, and transfers and subsidies were lower than expected owing to the late execution of some commitments or incorrect procurement procedures. With regard to capital expenditures, expenditures financed through external loans fell short of projections as a result of delays in the award of some contracts. Domestically-financed investments were in line with the program, except in the case of participation in the refinery, scheduled for 2008 but postponed to 2009. Taxes payable by the government on the execution of externally-financed projects were higher than expected. As a result of the significantly higher basic balance, the improvement in the government's net position vis-à-vis the central bank exceeded program targets by CFAF 50 billion or 2 percent of GDP.

\section{Macroeconomic prospects for 2009}

7. As a result of the possible dip in agricultural production following the 2008 record levels, GDP growth is expected to be about 3 percent in 2009. Uranium production is set to rise by 13 percent, fuelled by recent large investments. Both public and private investments 
are expected to increase sharply as construction begins on the Kandaji dam and work proceeds on the roadworks projects launched in 2008 as well as on construction of the new oil refinery in Zinder, the new uranium mines in Imouraren and Azelik, and the very ambitious drilling program at the Agadem oil field. Thus, the investment-to-GDP ratio could rise to around 36 percent. Private consumption is likely to be pushed up by the large upturn in farmers' incomes in 2008, with knock-on effects on the import of consumer goods. Yearon-year inflation is projected to fall to 2 percent, and the average annual rate to 5 percent. Estimates put the current account deficit at 24 percent of GDP and foreign reserves attributed to Niger could decline slightly.

\section{Budget execution in 2009 and fiscal reforms}

8. Tax revenues are projected to exceed the original program forecasts, reaching CFAF 299 billion (11.7 percent of GDP), of which CFAF 107 billion from customs and CFAF 192 billion from the General Tax Directorate (DGI). Revenues from profit tax on mining companies are set to increase by CFAF 17 billion, arising from SOPAMIN's (the uranium state marketing company) high profit in 2008, resulting from the resale at the spot price of 830 metric tons of uranium. This profit level is likely to be eroded in 2009, with the attendant impact on taxes payable in 2010. On the expenditure side, the revised forecasts are consistent with the 2009 Budget Law and take account of a realistic execution rate for investment expenditure, below 100 percent in some sectors. Government investment in the refinery, amounting to CFAF 19.5 billion, or 0.8 percent of GDP, and originally programmed in 2008, will take place in 2009 as reflected in the Budget Law. Consequently, the basic deficit should reach CFAF 148 billion, or 5.8 percent of GDP, compared with CFAF 134 billion, or 5.2 percent of GDP, originally programmed.

9. External budgetary support is projected to reach CFAF 92 billion, i.e., 3.6 percent of GDP, up markedly from CFAF 36 billion or 1.5 percent of GDP in 2008, and close to program projections. The increase from 2008 is due to the postponement to 2009 of European Union and World Bank disbursements, initially scheduled for 2008, as well as of support under the ten-year development plan for education. Domestic banking sector financing is projected at CFAF 79 billion (3.1 percent of GDP), compared to CFAF 61 billion under the initial program, and will take the form of draw down of central bank deposits. A CFAF 10 billion bond issue is planned in the first half of the year to capitalize Finaposte (CFAF 5 billion) and partially repay the statutory advance from the central bank.

10. In 2009, efforts to strengthen public financial management will focus on the following elements in the context of the PEMFAR agreed with donors: (i) strengthening computer linkages between the Budget and Treasury Directorates; (ii) computerization of decentralized budget offices (centres de sous-ordonnancements) and installation of appropriate software for reporting data on the execution of delegated appropriations; (iii) increasing the number of financial comptrollers from 13 to 33-appointments are in 
progress; (iv) enhancing the skills of the heads of procurement divisions of the various ministries; (v) organizing the structure of the Audit Office, (vi) connecting the customs border offices to the main regional centers for more efficient monitoring of trade flows; (vii) preparing tax reforms at the DGI aimed particularly at eliminating the ceiling on VAT credit refunds for exporters, and reviewing the real estate tax to ensure that it is not applied to production tools; and (viii) conducting a comprehensive review of the DGI's IT procedures to make them more effective.

11. With regard to budget preparation, a medium-term public expenditure framework is currently under preparation to support the 2010 budget and facilitate presentation of the budget in a multi-year framework that takes account of the priority objectives of the Growth and Poverty Reduction Strategy (SDRP) as well as of the constraints related to domestic resources and available external assistance. The IMF Fiscal Affairs Department is providing technical assistance to strengthen national capacities in this area.

12. The permanent secretariat of the SDRP launched the process of preparing the report on implementation of the SDRP in 2008, convening meetings of sectoral groups. The report is expected to be ready by next July to provide input for the budgetary discussions on the 2010 budget.

\section{Financial sector reforms}

13. Substantial progress is being made in modernizing the financial system. All of the commercial banks have committed to comply with the Banking Commission's new CFAF 5 billion minimum capital requirement ahead of the end-2010 deadline. Two of the ten banks are already in compliance. BIA, the second largest bank in terms of deposit amounts, is set to reach agreement shortly with a strategic investor for the takeover of a significant share of its capital previously held by a major European bank. The government will execute a short term portage operation pursuant to the agreement with the bank's Board of Directors in early February 2009. Non performing loans fell from 21.8 percent at end-2007 to 16.4 percent at end-2008 as recovery efforts produced results. In response to the strong demand for medium-term loans, banks expressed interest in higher availability of medium-term credit lines from external multilateral and bilateral institutions. A new EIB credit line of Euro 8 million is likely to be rapidly exhausted. Some regional retail banks are exploring the possibility of bond issues on the regional market to increase their long-term funds.

14. The government is determined to establish an agricultural bank. However, the key technical aspects - such as number of agencies and location, and the interface with existing banks - are still being carefully studied. Given the technical difficulties involved in ensuring the success of this new initiative, the design of the bank's operating arrangements will be worked out through in-depth analysis. 
15. Regarding the establishment of FINAPOSTE, the postal bank, an agreement for the issuance of non-negotiable treasury securities on behalf of the new entity is to be finalized shortly. Mobilization, in the coming months, of the minimum required capital of CFAF 5 billion should clear the way for its licensing by the Banking Commission at its June session.

16. With respect to microfinance, the microfinance regulatory agency is about to start up operations, and progress in strengthening various microfinance networks, including Taimako, UMEC, and MCPEC, has been encouraging.

\section{Management of petroleum and mining sector investments}

17. The government will incur significant obligations in the Agadem oil field drilling program. It holds a 20 percent share in the field, of which 15 percent is in portage by the exploration company and the remaining 5 percent includes an obligation to contribute directly to drilling expenditures. As the oil field's exploration and development programs provide for expenditures of around US\$500 million a year on average between 2009 and 2011, this translates into disbursements for the government of around US\$25 million a year on average, which will be provided for in the state budget.

18. With reference to the Zinder refinery, potentially profitable outlets for refined petroleum products need to be quickly identified in neighboring countries, particularly Nigeria, which has considerable absorptive capacity, as local demand is expected to consume only one third of the total output of these products. Since the government's 40 percent share of the refinery construction costs is to be recouped from future profits, with the exception of the 2009 initial budget contribution, it is vital for the refinery to be sufficiently profitable.

19. The government's 33 percent share in the Azelik uranium mine operating company entails the obligation to bear one-third of investment expenditures, which could reach US\$300 million. The government is negotiating a concessional loan with the Export -Import Bank of China to cover its capital contribution to the mine, and will consult closely with the Fund staff on this negotiation.

\section{Program monitoring}

20. The end- June and end-December 2009 quantitative performance criterion on domestic government financing has been modified to take account of the updated projections for public investment and external aid, and the government is seeking approval of the changes as shown in attached Table 1 . The two structural performance criteria originally set for end-2009 have now been transformed into benchmarks, following the changes in Fund policy in this area; the implementation of the related measures will be an important element of the fourth review, and we remain strongly committed to their implementation. The attached Technical Memorandum of Understandings of December 3, 2008 remains virtually 
unchanged from that of December 3, 2008 except for the modification of the adjustor in paragraph 9 from CFAF 30 billion to CFAF 35 billion. The list of structural benchmarks for 2009 is presented in Table 2 attached.

21. The government of Niger requests the conclusion of the second review under the PRGF-supported program. As in the past, the government authorizes the IMF to publish this Letter of Intent, and the IMF staff report. The government believes that the policies set out in this Letter of Intent are adequate to achieve the objectives of its program and will take any additional measures that may be necessary to that end. Niger will consult with the IMF on the adoption of such measures consistent with the policies of the IMF on these consultations.

Yours truly,

/s/

Ali Lamine Zeine

Minister of Economy and Finance

Attachment:

Technical Memorandum of Understandings 
Table 1a. Niger: Quantitative Performance Criteria and Indicative Targets, January 1, 2008-December 31, 2008 (Billions of CFA francs)

\begin{tabular}{|c|c|c|c|c|c|c|c|c|c|c|c|c|}
\hline & \multicolumn{3}{|c|}{$\begin{array}{c}\text { End-March } \\
\text { Indicative Targets }\end{array}$} & \multicolumn{3}{|c|}{$\begin{array}{l}\text { End-June } \\
\text { Performance Criteria }\end{array}$} & \multicolumn{3}{|c|}{$\begin{array}{l}\text { End-September } \\
\text { Indicative Targets }\end{array}$} & \multicolumn{3}{|c|}{$\begin{array}{c}\text { End-December } \\
\text { Performance Criteria }\end{array}$} \\
\hline & 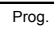 & Prog. Adj. & Est. & Prog. & 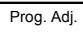 & Est. & 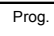 & $\begin{array}{c}\text { Prog. Adj. } \\
\text {. }\end{array}$ & 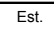 & Prog. & $\begin{array}{l}\text { Prog. Adj. } \\
\text {. }\end{array}$ & Est. \\
\hline \multicolumn{13}{|l|}{$\begin{array}{l}\text { A. Quantitative performance criteria and indicative targets } \\
\text { (cumulative from December } 31,2007 \text { ) }\end{array}$} \\
\hline Reduction in government domestic payments arrears ${ }^{3}$ & $\begin{array}{l}1.4 \\
4.0\end{array}$ & $\begin{array}{l}12.4 \\
4.0\end{array}$ & $\begin{array}{c}3.6 \\
-2.1\end{array}$ & $\begin{array}{l}18.1 \\
7.0\end{array}$ & $\begin{array}{l}21.5 \\
7.0\end{array}$ & $\begin{array}{l}-126.3 \\
-5.7\end{array}$ & $\begin{array}{c}38.7 \\
8.0\end{array}$ & $\begin{array}{l}49.0 \\
8.0\end{array}$ & $\begin{array}{l}-90.3 \\
-11.2\end{array}$ & $\begin{array}{l}-9.3 \\
15.2\end{array}$ & $\begin{array}{c}-6.8 \\
15.2\end{array}$ & $\begin{array}{l}-51.2 \\
-15.7\end{array}$ \\
\hline \multicolumn{13}{|l|}{ Memorandum item: } \\
\hline $\begin{array}{l}\text { Exceptional external budgetary assistance }{ }^{4} \\
\text { Gross budget support }\end{array}$ & $\begin{array}{l}13.5 \\
16.0\end{array}$ & & $\begin{array}{l}2.5 \\
5.0\end{array}$ & $\begin{array}{l}24.5 \\
29.6\end{array}$ & $\ldots$ & $\begin{array}{l}21.1 \\
25.2\end{array}$ & $\begin{array}{l}33.7 \\
41.4\end{array}$ & $\ldots$ & $\begin{array}{l}23.4 \\
28.4\end{array}$ & $\begin{array}{l}29.6 \\
38.9\end{array}$ & $\ldots$ & $\begin{array}{l}27.1 \\
35.6\end{array}$ \\
\hline Debt service & 2.6 & & 2.5 & 5.2 & & 4.2 & 7.7 & & 5.0 & 9.3 & $\ldots$ & 8.5 \\
\hline \multicolumn{13}{|l|}{ B. Continuous quantitative performance criteria } \\
\hline $\begin{array}{l}\text { Accumulation of external payments arrears } \\
\text { New external debt contracted or guaranteed }\end{array}$ & 0.0 & $\ldots$ & 0.0 & 0.0 & $\ldots$ & 0.0 & 0.0 & & 0.0 & 0.0 & & 0.0 \\
\hline $\begin{array}{l}\text { by the government with maturities of } 0-1 \text { year } \\
\text { New nonconcessional external debt contracted or guaranteed } \\
\text { by the government with maturities over } 1 \text { year : }\end{array}$ & 0.0 & & 0.0 & 0.0 & $\ldots$ & 0.0 & 0.0 & & 0.0 & 0.0 & $\ldots$ & 0.0 \\
\hline grant element lower than 35 percent & 0.0 & & 0.0 & 0.0 & & 0.0 & 0.0 & & 0.0 & 0.0 & & 0.0 \\
\hline \multicolumn{13}{|l|}{$\begin{array}{l}\text { C. Indicative Targets } \\
\text { (cumulative from December } 31,2007 \text { ) }\end{array}$} \\
\hline $\begin{array}{l}\text { Basic budget balance (commitment basis, excl. grants) } \\
\text { Total revenue }^{7}\end{array}$ & $\begin{array}{l}-11.8 \\
83.9 \\
0.0\end{array}$ & & $\begin{array}{c}3.5 \\
81.7 \\
0.0\end{array}$ & $\begin{array}{r}-42.6 \\
148.8\end{array}$ & $\begin{array}{l}\ldots \\
\ldots\end{array}$ & $\begin{array}{c}112.6 \\
356.4 \\
0.0\end{array}$ & $\begin{array}{l}-72.4 \\
214.7\end{array}$ & $\begin{array}{l}\ldots \\
\ldots\end{array}$ & $\begin{array}{c}77.7 \\
356.4 \\
0.0\end{array}$ & $\begin{array}{l}-14.9 \\
407.4\end{array}$ & $\begin{array}{l}\ldots \\
\ldots\end{array}$ & $\begin{array}{r}30.9 \\
442.0 \\
0.0\end{array}$ \\
\hline
\end{tabular}

Note: The terms in this table are defined in the TMU.

"Performance criteria for program indicators under $\mathrm{A}$ and $\mathrm{B}$; indicative targets otherwise.

Shursements of external budgetary assistance, as defined in footnote 4 , exceeds or falls short of program forecasts

The

${ }^{3}$ Minimum.

${ }^{4}$ External budgetary assistance (including traditional debt relief, HIPC Initititive assistance, but excluding net financing from the IMF) less external debt sevvice and payments of external arrears.

${ }^{5}$ Excluding ordinary credit for imports or debt relief.

Exher

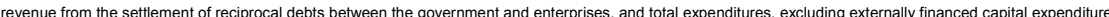

${ }^{8}$ Minimum. Excluding (i) revenue from the settlement of reciprocal debts between the government and Nigerien enterprises; and (ii) revenue from the privatization of public enterprises that is included in financing. 
Table 1b. Niger: Quantitative Performance Criteria and Indicative Targets, January 1, 2009-December 31, 2009

(Billions of CFA francs)

\begin{tabular}{|c|c|c|c|c|c|c|c|}
\hline & \multirow{2}{*}{$\begin{array}{r}\text { End-March } \\
\text { Indicative Targets } \\
\text { Init. Prog. }\end{array}$} & \multicolumn{2}{|c|}{$\begin{array}{c}\text { End-June } \\
\text { Performance Criteria }\end{array}$} & \multicolumn{2}{|c|}{$\begin{array}{l}\text { End-September } \\
\text { Indicative Targets }\end{array}$} & \multicolumn{2}{|c|}{$\begin{array}{c}\text { End-December } \\
\text { Performance Criteria }\end{array}$} \\
\hline & & Init. Prog. & Rev. Prog. & Init. Prog. & Rev. Prog. & Init. Prog. & Rev. Prog. \\
\hline Domestic financing of the budget ${ }^{1,2}$ & 12.1 & 62.5 & 54.8 & 67.2 & 83.4 & 55.9 & 74.5 \\
\hline Reduction in government domestic payments arrears ${ }^{3}$ & -2.0 & -6.0 & -6.0 & -8.0 & -8.0 & -15.0 & -15.0 \\
\hline \multicolumn{8}{|l|}{ Memorandum item: } \\
\hline Exceptional external budgetary assistance ${ }^{4}$ & 20.6 & 5.7 & 29.8 & 37.9 & 34.0 & 86.6 & 82.3 \\
\hline Gross budget support & 23.0 & 10.5 & 34.4 & 45.2 & 41.0 & 95.7 & 91.6 \\
\hline Debt service & 2.4 & 4.8 & 4.7 & 7.3 & 7.0 & 9.7 & 9.3 \\
\hline \multicolumn{8}{|l|}{ B. Continuous quantitative performance criteria } \\
\hline Accumulation of external payments arrears & 0.0 & 0.0 & 0.0 & 0.0 & 0.0 & 0.0 & 0.0 \\
\hline \multicolumn{8}{|l|}{ New external debt contracted or guaranteed } \\
\hline by the government with maturities of $0-1$ year ${ }^{5}$ & 0.0 & 0.0 & 0.0 & 0.0 & 0.0 & 0.0 & 0.0 \\
\hline \multicolumn{8}{|l|}{$\begin{array}{l}\text { New nonconcessional external debt contracted or guaranteed } \\
\text { by the government with maturities over } 1 \text { year }{ }^{6} \text { : }\end{array}$} \\
\hline \multicolumn{8}{|l|}{$\begin{array}{l}\text { C. Indicative Targets } \\
\text { (cumulative from December } 31,2008 \text { ) }\end{array}$} \\
\hline Basic budget balance (commitment basis, excl. grants) ${ }^{7}$ & -30.3 & -66.9 & -83.3 & -101.0 & -115.3 & -133.6 & -148.5 \\
\hline Total revenue $^{8}$ & 79.3 & 152.3 & 147.1 & 227.8 & $230.3 \#$ & 304.8 & 312.3 \\
\hline
\end{tabular}

Note: The terms in this table are defined in the TMU. Note that for End-March indicative targets, the figure are those of the initial program, and not those projected in the Quarterly fiscal table $2 c$

'Performance criteria for program indicators under A and B; indicative targets otherwise. Domestic financing in this table excludes IMF financing-TMU.

The ceiling on domestic financing of the budget will be adjusted if the amount of disbursements of external budgetary assistance, as defined in footnote 4 , falls short of program forecasts

If disbursements are less than the programmed amounts, the ceiling will be raised pro tanto, up to a maximum of CFAF 35 billion at the end of each quarter of 2009.

${ }^{3}$ Minimum.

${ }^{4}$ External budgetary assistance (including traditional debt relief, HIPC Initiative assistance, but excluding net financing from the IMF) less external debt service and payments of external arrears.

${ }^{5}$ Excluding ordinary credit for imports or debt relief.

${ }^{6}$ Excluding debt relief obtained in the form of rescheduling or refinancing

${ }^{7}$ Minimum, defined as the difference between total revenue, excluding grants and revenue from the settlement of reciprocal debts between the government and enterprises, and total expenditures, excluding externally financed capital expenditures.

${ }^{8}$ Minimum. Excluding (i) revenue from the settlement of reciprocal debts between the government and Nigerien enterprises; and (ii) revenue from the privatization of public enterprises that is included in financing 


\section{Table 2. Niger: Structural Benchmarks for the Program December 2008-December-2009}

\section{Measures}

\section{Structural benchmarks}

Presentation in the budget law for 2009 of the investment programs for the priority sectors of the PRSP for 2009-2012.

Production by the Ministry of Finance of semiannual reports on the foreign debt contracted and its terms, and on the borrowing program for the next six months and the terms specified.

Issue data on budget outturn for 2008, and for 2009 on a quarterly basis, including for the unified list of priority expenditures and the President's Special Program.

Adoption by the Council of Ministers of the MTEF for the infrastructure and transport sectors.

Reduction of the fees for registering a new business in the Register of Commerce at the courts.

The Budget Law for 2010 will include a production of the main budget aggregates (revenue and expenditure) for the period 2010-12.

Elimination of the ceiling for reimbursement of VAT credits to all exporters (originally set as performance criterion).

Reduction of the rate of profit tax from 35 to 30 percent, applicable to profits reported for FY 2009 and for following years (originally set as performance criterion).
End-December 2008

Not met

End-December 2008 and

Met

the end of each

successive half-year

period

End-March 2009 for end 2008 budgetary outturn, and end of each 2009 quarter for quarterly 2009 data

End- June 2009

End-June 2009

Met

End-December 2009

End-December 2009

Budgetary execution data for end-2008 issued

End-December 2009 


\section{TeChNiCAL Memorandum OF Understanding}

Niamey, April 27, 2009

1. This technical memorandum of understanding defines the performance criteria and indicative targets for Niger's program under the Poverty Reduction and Growth Facility (PRGF) for the period 2008-11. The performance criteria and indicative targets for end- endJune and end-December 2009 are set out in Table 1 of the government's Memorandum of Economic and Financial Policies (MEFP) dated April 27, 2009 and attached hereto. This technical memorandum of understanding also sets out data-reporting requirements for monitoring the program.

\section{DEFINITION OF TERMS}

2. For the purpose of this technical memorandum, the following definitions of "debt," "government," "payments arrears," and "government obligations" will be used:

(a) As specified in point 9 of the Guidelines on Performance Criteria with Respect to Foreign Debt adopted by the Executive Board of the IMF on August 24, 2000 , debt will be understood to mean a current, that is, not contingent, liability, created under a contractual arrangement through the provision of value in the form of assets (including currency) or services, and which requires the obligor to make one or more payments in the form of assets (including currency) or services, according to a specific schedule; these payments will discharge the obligor of the principal and/or interest liabilities incurred under the contract. Debts can take a number of forms, the primary ones being as follows: (i) loans, that is, advances of money to the obligor by the lender made on the basis of an undertaking that the obligor will repay the funds in the future (including deposits, bonds, debentures, commercial loans, and buyers' credits) and temporary exchanges of assets that are equivalent to fully collateralized loans, under which the obligor is required to repay the funds, and usually pay interest, by repurchasing the collateral from the buyer in the future (such as repurchase agreements and official swap arrangements); (ii) suppliers' credits, that is, contracts where the supplier permits the obligor to defer payments until some time after the date on which the goods are delivered or services are provided; and (iii) leases, that is, arrangements under which property is provided that the lease holder has the right to use for one or more specified period(s) of time that are usually shorter than the total expected service life of the property, while the lease grantor retains the title to the property. For the purpose of this guideline, the debt is the present value (at the inception of the lease) of all lease payments expected to be made during the period of the agreement, excluding those payments necessary for the operation, repair, or maintenance of the property. Under the definition of debt set out above, arrears, penalties, and judicially awarded damages arising from the failure to make payment under a contractual obligation that constitutes debt are debt. Failure to make payment on an obligation that is not considered debt under this definition (for example, payment on delivery) will not give rise to debt. 
(b) Government refers to the central government of the Republic of Niger; it does not include any political subdivision, public entity, or central bank with a separate legal personality.

(c) External payment arrears are external payments due but not paid. Domestic payment arrears are domestic payments due but not paid. They include (i) arrears outstanding at end-1999 identified by the audit conducted by the Ministry of Finance in 2005; and (ii) the reste à payer at the Treasury related to the budgetary years 2004, 2005, 2006, and 2007 remaining due at December 31, 2008.

(d) Government obligation is any financial obligation of the government accepted as such by the government (including any government debt).

\section{Quantitative Performance Criteria}

\section{A. Net Domestic Financing of the Government}

\section{Definition}

3. Net domestic financing of the government is defined as the sum of (i) net bank credit to the government, as defined below; and (ii) net nonbank domestic financing of the Government, including government securities issued in CFA francs on the WAEMU regional financial market and not held by resident commercial banks, proceeds from the sale of government assets, and privatization receipts net of the cost of structural reforms to which these proceeds are earmarked.

4. Net bank credit to the government is equal to the balance of the government's claims and debts vis-à-vis national banking institutions. Government claims include cash holdings by the Nigerien Treasury, deposits with the central bank and commercial banks, and secured obligations. Government debt to the banking system includes debt vis-à-vis the central bank (excluding net financing from the IMF's Poverty Reduction and Growth Facility (PRGF), but including government securities) and to commercial banks (including government securities held by commercial banks), and deposits with the postal checking system.

5. The scope of the net bank credit to the government as defined by the BCEAO includes all central government administrations. Net bank credit to the government and the amounts of government treasury bills and bonds issued in CFA francs on the regional financial market of the WAEMU are calculated by the BCEAO, and the net nonbank financing of the government is calculated by the Nigerien Treasury, whose figures are those deemed valid within the context of the program. 
6. Nonbank net domestic financing includes (i) the change in the amount of government bonds issued in the regional WAEMU market and not held by Niger's commercial banks; (ii) the change in the deposits of Treasury correspondents; and (iii) the change in "comptes de consignations" at the Treasury.

7. The 2009 quarterly targets are based on the change in stock between end-December 2008 and the date considered for the performance criterion or the indicative target.

\section{Adjustment}

8. The ceiling on net domestic financing will be subject to adjustments if disbursements of external budgetary support less external debt service and arrears payments, including disbursements under the PRGF, fall short of projected amounts. For 2009, external budget support is calculated from end-December 2008.

9. If disbursements fall short of projected external budgetary assistance for each quarter in 2009, the corresponding quarterly ceilings on net domestic financing will be raised pro tanto, up to a maximum of CFAF 35 billion.

\section{Reporting requirement}

10. Detailed data on domestic financing to government will be provided monthly within six weeks after the end of each month.

\section{B. Reduction of Domestic Payments Arrears}

\section{Definition}

11. Domestic payments arrears comprise (i) arrears identified at end-1999 on the basis of the audit conducted by the Ministry of Finance in 2005; and (ii) the reste à payer(RAP) at the Treasury for budget years 2004, 2005, 2006 and 2007 outstanding at end-2008. The stock of arrears will be reduced to the minimum of the amounts indicated in Table 1 annexed to the MEFP. The quarterly objectives for 2009 are based on the changes in the stock of arrears from end-December 2008, and the date selected for the performance criterion or indicative target. The stock of RAP at end-2009 for the 2009 budget year will not exceed the stock of RAP outstanding at end- 2008 for the 2008 budget year; any excess will be considered an increase in arrears, that will be deducted from the reduction of arrears as defined as the beginning of this paragraph. 
12. The Centre d'Amortissement de la Dette Intérieure de l'Etat (CADDIE) and the Treasury are responsible for calculating the stock of domestic arrears, and recording their repayments.

\section{Reporting requirement}

13. Monthly data on the outstanding balance, accumulation (including changes in the reste à payer at the Treasury), and repayment of domestic payments arrears on government obligations will be provided monthly within six weeks following the end of each month.

\section{Reduction of External Payments Arrears}

\section{Definition}

14. Government debt is outstanding debt owed or guaranteed by the government. For the program, the government undertakes not to accumulate external arrears on government debt (including treasury bills and bonds issued in CFA francs on the WAEMU regional financial market), with the exception of external payments arrears arising from government debt being renegotiated with external creditors, including Paris Club creditors.

\section{Reporting requirement}

15. Data on the outstanding balance, accumulation, and repayment of external payments arrears will be provided monthly within six weeks following the end of each month.

\section{External Nonconcessional Loans Contracted or Guaranteed by the Government of Niger}

\section{Definition}

16. The government will not contract or guarantee external debt with original maturity of one year or more with a grant element of less than 35 percent. Nonconcessional external debt is defined as all debt with a concessionality level of less than 35 percent. To calculate the level of concessionality for loans with a maturity of at least 15 years, the discount rate to be used is the 10-year average commercial interest reference rate (CIRR), calculated by the IMF on the basis of the rates published by the OECD; for loans of less than 15 years, the six-month average CIRR is to be used. The Ministry of Finance will communicate regularly to Fund staff the list of loans under negotiations, and, in case of objections, the Fund staff will have to express any objections within two weeks.

17. This performance criterion applies not only to debt as defined in point 9 of the Guidelines on Performance Criteria with Respect to Foreign Dept adopted by the Executive Board on August 24, 2000, but also to commitments contracted or guaranteed for which value has not been received. However, this performance criterion does not apply to financing provided by the Fund, to debt rescheduling in the form of new loans, and to treasury notes and bonds issued in CFA francs on the WAEMU regional financial market. 


\section{Reporting requirement}

18. Details on any external government debt will be provided monthly within six weeks after the end of each month. The same requirement applies to guarantees extended by the central government.

\section{E. Short-Term External Debt of the Central Government}

\section{Definition of the performance criterion}

19. The government will not accumulate or guarantee new external debt with original maturity of less than one year. This performance criterion applies not only to debt as defined in point 9 of the Guidelines on Performance Criteria with Respect to Foreign Debt adopted on August 24, 2000, but also to commitments contracted or guaranteed for which value has not been received. Excluded from this performance criterion are short-term, import-related trade credits and short-term treasury notes issued in CFA francs on the regional financial market.

\section{Reporting requirement}

20. Details on any external government debt will be provided monthly within six weeks following the end of each month. The same requirement applies to guarantees extended by the central government.

\section{Quantitative TARgets}

\section{A. Definitions}

21. Total revenue is an indicative target for the program. It includes tax, nontax, and special accounts revenue, but excludes revenue from the settlement of reciprocal debts between the government and enterprises.

22. The basic fiscal deficit is defined as the difference between: (i) total fiscal revenue as defined in paragraph 23; and (ii) total fiscal expenditure excluding foreign financed investment (but including HIPC-financed investment).

23. This information will be provided to the IMF monthly within six weeks after the end of each month. 


\section{Additional Information for Program-Monitoring Purposes}

\section{A. Public Finances}

24. The government will report to IMF staff the following:

- detailed monthly estimates of revenue and expenditure, including priority expenditure, the payment of domestic and external arrears, and a breakdown of customs, DGI, and Treasury revenue;

- the table of government financial operations with comprehensive monthly data on domestic and external financing, and the changes in arrears (arrears outstanding at end-1999) and reste à payer (RAP) at the Treasury. These data are to be provided monthly within six weeks following the end of each month;

- quarterly data on expenditures of the unified priority list, and data on expenditures on HIPC resources and the President's Special Program, on a payment order basis;

- quarterly data on implementation of the Public Investment Program, including details on financing sources, to be provided quarterly within eight weeks following the end of each quarter;

- monthly data on the balances of the accounts of the Treasury and of other public accounting officers at the BCEAO;

- monthly data on the reste à payer at the Treasury, by reference fiscal year with an itemization of maturities of more than, and less than, 120 days;

- monthly data on effective debt service (principal and interest) compared with the planned schedules. These data are to be provided within four weeks after the end of each month.

\section{B. Monetary Sector}

25. The government will provide the following information within eight weeks following the end of each month:

- the consolidated balance sheet of monetary institutions and, as appropriate, the balance sheets of selected individual banks;

- the monetary survey within eight weeks after the end of the month (provisional data);

- borrowing and lending interest rates; and

- customary banking supervision indicators for bank and nonbank financial institutions (if necessary, the same indicators for individual institutions may also be provided).

\section{Balance of Payments}

26. The government will provide IMF staff with the following information:

- any revision to balance of payments data (including services, private transfers, official transfers, and capital transactions) whenever they occur; and

- preliminary annual balance of payments data, within six months after the end of the year concerned. 


\section{Real Sector}

27. The government will provide IMF staff with the following information:

- disaggregated monthly consumer price indices, monthly within two weeks following the end of each month;

- national accounts, within six months after the end of the year; and

- any revision in the national accounts.

\section{E. Structural Reforms and Other Data}

28. The government will provide the following information:

- any study or official report on Niger's economy, within two weeks after its publication; and

- any decision, order, law, decree, ordinance, or circular with economic or financial implications, upon its publication or, at the latest, when it enters into force. 
Summary of Main Data Requirements

\begin{tabular}{|c|c|c|c|}
\hline Type of Data & Tables & Frequency & $\begin{array}{l}\text { Reporting } \\
\text { Requirement }\end{array}$ \\
\hline \multirow[t]{3}{*}{ Real sector } & National accounts & Annual & $\begin{array}{l}\text { End of year }+ \text { six } \\
\text { months }\end{array}$ \\
\hline & Revisions of national accounts & Irregular & $\begin{array}{l}\text { Eight weeks } \\
\text { following revision }\end{array}$ \\
\hline & Consumer price indexes, disaggregated & Monthly & $\begin{array}{l}\text { End of month + two } \\
\text { weeks }\end{array}$ \\
\hline \multirow[t]{8}{*}{$\begin{array}{l}\text { Public } \\
\text { finances }\end{array}$} & $\begin{array}{l}\text { Net government position in the banking } \\
\text { sector }\end{array}$ & Monthly & $\begin{array}{l}\text { End of month }+ \text { six } \\
\text { weeks }\end{array}$ \\
\hline & $\begin{array}{l}\text { Provisional table of government } \\
\text { financial operations, including } \\
\text { breakdown of revenue (DGI, DGD, and } \\
\text { Treasury) and expenditure, including } \\
\text { repayments of domestic wage and non- } \\
\text { wage arrears outstanding at end-1999 } \\
\text { and the change in the reste à payer } \\
\text { (RAP) at the Treasury. }\end{array}$ & Monthly & $\begin{array}{l}\text { End of month }+ \text { six } \\
\text { weeks }\end{array}$ \\
\hline & $\begin{array}{l}\text { Data on the stock of reste à payer at } \\
\text { the Treasury, by reference fiscal year } \\
\text { (total and RAP older than } 120 \text { days) }\end{array}$ & Monthly & $\begin{array}{l}\text { End of month }+ \text { six } \\
\text { weeks }\end{array}$ \\
\hline & $\begin{array}{l}\text { Monthly data on the deposits of the } \\
\text { correspondents with the Treasury }\end{array}$ & Monthly & $\begin{array}{l}\text { End of month }+ \text { six } \\
\text { weeks }\end{array}$ \\
\hline & Investment expenditure execution & Quarterly & $\begin{array}{l}\text { End of quarter + eight } \\
\text { weeks }\end{array}$ \\
\hline & $\begin{array}{l}\text { Table of execution of budgetary } \\
\text { expenditures, of the expenditures in the } \\
\text { priority unified list, and of expenditures } \\
\text { on HIPC resources }\end{array}$ & Quarterly & $\begin{array}{l}\text { End of quarter + six } \\
\text { weeks }\end{array}$ \\
\hline & General balance of Treasury accounts & Monthly & $\begin{array}{l}\text { End of month + six } \\
\text { weeks }\end{array}$ \\
\hline & $\begin{array}{l}\text { Monthly data on Treasury account } \\
\text { balances and other public entities at the } \\
\text { BCEAO. }\end{array}$ & Monthly & $\begin{array}{l}\text { End of month + two } \\
\text { weeks }\end{array}$ \\
\hline
\end{tabular}




\title{
INTERNATIONAL MONETARY FUND
}

NIGER

\section{Second Review Under the Three-Year Arrangement Under the Poverty Reduction and} Growth Facility and Request for Modification of Performance Criteria

\author{
Informational Annex \\ Prepared by the African Department \\ (In consultation with other departments) \\ Approved by Tom Krueger and Anthony Boote
}

April 30, 2009

- $\quad$ Relations with the Fund. Describes financial and technical assistance by the IMF, and provides information on the exchange rate system. Niger's PRGF arrangement was approved on May 28, 2008.

- IMF-World Bank Joint Monitoring Action Plan. Describes the World Bank Group's strategy and portfolio. 


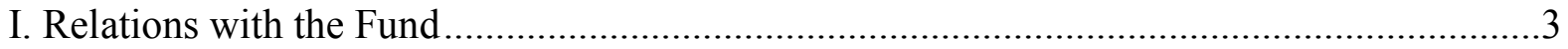

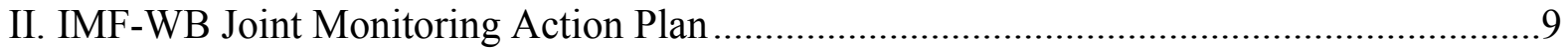

A. Mutual Information on Relevant Work Programs ................................................ 9

B. Requests for Work Program Inputs (as needed).....................................................

C. Agreement on Joint Products and Missions (as needed) ...................................... 9

III. Table of Common Indicators Required for Surveillance ..............................................11 


\section{RELATIONS WITH THE FUND}

(As of March 31, 2009)

I. Membership Status: Joined: April 24, 1963;

Accepted Obligations of Article VIII, Sections 2, 3 and 4: June 1, 1996

II. General Resources Account:

SDR Million $\quad \%$ Quota

Quota

65.80

100.00

Fund Holdings of Currency

57.19

86.92

Reserve Position

8.61

13.09

Holdings Exchange Rate

\section{SDR Department:}

Net Cumulative Allocation

Holdings

IV. Outstanding Purchases and Loans:

PRGF Arrangements

$\begin{array}{cc}\text { SDR Million } & \text { \%Allocation } \\ 9.41 & 100.00 \\ 1.05 & 11.21\end{array}$

SDR Million

29.61

\%Quota

45.00

\section{Latest Financial Arrangements:}

$\begin{array}{lcccc}\text { Type } & \begin{array}{c}\text { Date of } \\ \text { Arrangement }\end{array} & \begin{array}{c}\text { Expiration } \\ \text { Date }\end{array} & \begin{array}{c}\text { Amount Approved Amount Drawn } \\ \text { (SDR Million) }\end{array} & \begin{array}{c}\text { (SDR Million) } \\ \text { PRGF }\end{array} \\ \text { Jun 2, 2008 } & \text { Jun 1, 2011 } & 23.03 & 3.29 \\ \text { PRGF } & \text { Jan 31, 2005 } & \text { May 31, 2008 } & 26.32 & 26.32 \\ \text { PRGF } & \text { Dec 22, 2000 } & \text { Jun 30, 2004 } & 59.20 & 59.20\end{array}$

VI. Projected Payments to Fund ${ }^{1 /}$

(SDR Million; based on existing use of resources and present holdings of SDRs):

Principal

Charges/Interest

Total

\begin{tabular}{lllll}
\multicolumn{5}{c}{ Forthcoming } \\
\hline$\underline{2008}$ & $\underline{2009}$ & $\underline{2010}$ & $\underline{2011}$ & $\underline{2012}$ \\
$\underline{0.13}$ & $\underline{0.36}$ & $\underline{0.36}$ & $\underline{0.36}$ & $\underline{0.34}$ \\
$\underline{0.13}$ & $\underline{0.36}$ & 0.46 & $\underline{3.29}$ & $\underline{4.55}$
\end{tabular}

\footnotetext{
${ }^{1 /}$ When a member has overdue financial obligations outstanding for more than three months, the amount of such arrears will be shown in this section.
} 


\section{Implementation of HIPC Initiative:}

I. Commitment of HIPC assistance

Decision point date

Assistance committed

by all creditors (US\$ million) ${ }^{1 /}$

(SDR equivalent in millions)

Completion point date
Of which: IMF assistance (US\$ million)

Enhanced

Framework

Dec. 2000

663.10

Apr. 2004

\section{Disbursement of IMF assistance (SDR million)}

Assistance disbursed to the member

Interim assistance

Completion point balance

Additional disbursement of interest income ${ }^{2 /}$

Total disbursements

\footnotetext{
${ }^{1 /}$ Assistance committed under the original framework is expressed in net present value (NPV) terms at the completion point, and assistance committed under the enhanced framework is expressed in NPV terms at the decision point. Hence these two amounts can not be added.

${ }^{2 /}$ Under the enhanced framework, an additional disbursement is made at the completion point corresponding to interest income earned on the amount committed at the decision point but not disbursed during the interim period.
}

Decision point - point at which the IMF and the World Bank determine whether a country qualifies for assistance under the HIPC Initiative and decide on the amount of assistance to be committed.

Interim assistance - amount disbursed to a country during the period between decision and completion points, up to 20 percent annually and 60 percent in total of the assistance committed at the decision point (or 25 percent and 75 percent, respectively, in exceptional circumstances).

Completion point - point at which a country receives the remaining balance of its assistance committed at the decision point, together with an additional disbursement of interest income as defined in footnote 2 above. The timing of the completion point is linked to the implementation of pre-agreed key structural reforms (i.e., floating completion point). 


\title{
VIII. Implementation of Multilateral Debt Relief Initiative (MDRI):
}

I. MDRI-eligible debt (SDR million) ${ }^{1 /}$

Financed by: MDRI Trust

59.82

Remaining HIPC resources

17.73

II. Debt Relief by Facility (SDR million)

\author{
Delivery Date \\ January 2006
}

\begin{tabular}{rrr}
\multicolumn{3}{c}{ Eligible Debt } \\
\hline GRA & PRGF & Total \\
N/A & 77.55 & 77.55
\end{tabular}

\footnotetext{
The MDRI provides 100 percent debt relief to eligible member countries that qualified for the assistance. Grant assistance from the MDRI Trust and HIPC resources provide debt relief to cover the full stock of debt owed to the Fund as of end-2004 that remains outstanding at the time the member qualifies for such debt relief.
}

\section{Safeguards Assessments:}

The Central Bank of West African States (BCEAO) is the common central bank of the countries of the West African Economic and Monetary Union, which includes Niger. The most recent safeguards assessment of the BCEAO was completed on November 4, 2005. The assessment indicated progress has been made in strengthening the bank's safeguards framework since the 2002 assessment and identified some areas where further steps would help solidify it.

The BCEAO now publishes a full set of audited financial statements and improvements have been made to move financial reporting closer to International Financial Reporting Standards (IFRS). Furthermore, an internal audit charter has been put in place, mechanisms for improving risk management have been established, and follow up on internal and external audit recommendations has been strengthened.

The results of continuous safeguards monitoring indicate that while certain vulnerabilities remain in internal control systems and legal structure, there has been some progress in other areas, including through: (i) improving the external audit process by adopting a multi-year audit program; (ii) establishing an audit committee; (iii) expanding disclosures on financial positions of WAEMU countries with the Fund in the notes to the annual financial statements; and (iv) further strengthening of the effectiveness of the internal audit function.

\section{Exchange Arrangements:}

Niger is a member of the West African Economic and Monetary Union (WAEMU). The exchange system, common to all members of the WAEMU, is free of restrictions on the making of payments and transfers for current international transactions. The WAEMU's common currency, the CFA franc, was originally pegged to the French franc. On January 12, 1994, the CFA franc was devalued by 50 percent in foreign currency terms, and the exchange 
rate was adjusted from CFAF $50=\mathrm{F} 1$ to CFAF $100=\mathrm{F} 1$. Effective December 31, 1998, the parity was switched to the Euro at a rate of CFAF $655.96=$ EUR 1. On November 7, 2008, the rate of the CFA franc in SDR terms was SDR 1 = CFAF 764.25. The exchange arrangement of the WAEMU countries is classified as a conventional pegged arrangement.

\section{Article IV Consultation:}

Niger is on the 24-month consultation cycle. The last Article IV consultation discussions were held in Niamey in September-October 2006, and discussed by the Executive Board on December 20, 2006.

\section{Technical Assistance:}

\begin{tabular}{|c|c|c|c|}
\hline Dept. & $\begin{array}{l}\text { Type of } \\
\text { Assistance }\end{array}$ & Time of Delivery & Responsibility \\
\hline FAD & Resident expert & June 2001 to May 2003 & Assistance for tax administration \\
\hline FAD & Staff & May 2002 & $\begin{array}{l}\text { Public accounting and fiscal } \\
\text { operations table }\end{array}$ \\
\hline STA & Staff & January 2003 & Multisector statistical mission \\
\hline FAD & Staff & October 2003 & $\begin{array}{l}\text { Tax policy and revenue } \\
\text { administration }\end{array}$ \\
\hline AFRITAC W & Advisor & April 2004 & Tax administration \\
\hline AFRITAC W & Advisor & August 2004 & Customs administration \\
\hline AFRITAC W & Advisor & October-November 2004 & Tax administration \\
\hline STA & Staff & March 2005 & ROSC \\
\hline AFRITAC W & Advisor and expert & March 2005 & Customs administration \\
\hline AFRITAC W & Advisor & March 2005 & Macroeconomic statistics \\
\hline AFRITAC W & Advisor & March-April 2005 & Microfinance \\
\hline AFRITAC W & Advisor & May-June 2005 & Macroeconomic statistics \\
\hline FAD & Staff & September 2005 & $\begin{array}{l}\text { Tax policy and revenue } \\
\text { administration }\end{array}$ \\
\hline
\end{tabular}




\begin{tabular}{|c|c|c|c|}
\hline FAD & Expert & $\begin{array}{l}\text { September/October } 2006 \\
\text { and January/February } 2007\end{array}$ & $\begin{array}{l}\text { Tax policy and revenue } \\
\text { administration }\end{array}$ \\
\hline AFRITAC W & Advisor & October 2005 & Customs administration \\
\hline AFRITAC W & Advisor & March 2006 & Microfinance \\
\hline AFRITAC W & Advisor and expert & May 2006 & Macroeconomic statistics \\
\hline AFRITAC W & Advisor & May 2006 & Public debt management \\
\hline AFRITAC W & Advisor & June-July 2006 & Macroeconomic statistics \\
\hline AFRITAC W & Advisor & November 2006 & Customs administration \\
\hline AFRITAC W & Advisor & December 2006 & Macroeconomic statistics \\
\hline AFRITAC W & Advisor & January 2007 & Tax administration \\
\hline AFRITAC W & Advisor & February-March 2007 & Microfinance \\
\hline AFRITAC W & Advisor & June 2007 & Macroeconomic statistics \\
\hline AFRITAC W & Advisor and expert & June 2007 & Customs administration \\
\hline AFRITAC W & Advisor and expert & July 2007 & Tax administration \\
\hline AFRITAC W & Expert & September 2007 & Public expenditure management \\
\hline AFRITAC W & Advisor & November 2007 & Macroeconomic statistics \\
\hline AFRITAC W & Advisor & February 2008 & Tax administration \\
\hline AFRITAC W & Advisor & February 2008 & Statistics \\
\hline AFRITAC W & Advisor & February-March 2008 & Microfinance \\
\hline AFRITAC W & Advisor & March 2008 & Public expenditure management \\
\hline AFRITAC W & Advisor & March 2008 & External debt management \\
\hline AFRITAC W & Advisor & April 2008 & Customs administration \\
\hline AFRITAC W & Advisor & May-June 2008 & Public finance statistics \\
\hline AFRITAC W & Advisor & June 2008 & Real sector statistics \\
\hline
\end{tabular}




$\begin{array}{llll}\text { FAD } & \text { Staff } & \text { September 2008 } & \text { Tax policy } \\ \text { AFRITAC W } & \text { Advisor and expert } & \text { October 2008 } & \text { Customs/Tax administration } \\ \text { AFRITAC W } & \text { Advisor } & \text { October 2008 } & \text { Tax administration } \\ \text { AFRITAC W } & \text { Advisor } & \text { January 2009 } & \text { National account statistics } \\ \text { FAD } & \text { Staff } & \text { March 2009 } & \text { Medium-term budget }\end{array}$

\section{Resident Representative:}

Mr. Mario Zejan has taken the position of resident representative in Niger since mid-April 2009. 


\section{IMF/WB-Joint Monitoring ACtion Plan}

\begin{tabular}{cccc}
\hline Title & $\begin{array}{c}\text { Products } \\
\text { (For example) }\end{array}$ & $\begin{array}{l}\text { Provisional Timing of } \\
\text { Missions (if relevant) }\end{array}$ & $\begin{array}{c}\text { Expected } \\
\text { Delivery Date }\end{array}$ \\
\hline
\end{tabular}

\section{A. Mutual Information on Relevant Work Programs}

$\begin{array}{llll}\begin{array}{l}\text { Bank work } \\ \text { program in next } \\ \text { 12 months }\end{array} & \text { Growth DPL } & \text { December 2008 } & \text { March 2009 } \\ & \text { Growth } & & \text { June 2009 } \\ & \text { PEMFAR II } & \text { February 2009 } & \text { July 2009 } \\ & \text { ESW on Rural Finance } & \text { To be determined } & \text { June 2009 } \\ & \text { ESW on Political Economy of } & & \\ & \text { mining } & \text { April 2009 } \\ & \text { TA on MEF Capacity Building } & \text { March 2009 } \\ \text { IMF work } & \text { Staff visit/program review } & \text { March 2009 } \\ \text { program in next } & \text { Staff visit/program review } & \text { September } \\ 12 \text { months } & & \text { 2009 }\end{array}$

\section{B. Requests for Work Program Inputs (as needed)}

Fund request to Bank (with

PER top-up to provide quantitative summary justification) inputs for budget framework

Analysis of supply-side implications of sectoral composition of aid

ESW on Trade (DTIS)

Bank request to Fund (with summary Assessment letter Macroeconomic scenarios associated justification) with scaling up of aid

\section{Agreement on Joint Products and Missions (as needed)}

Joint products in Debt sustainability analysis

December next 12 months 


\section{List of acronyms}

DPL Development Policy Loan

DTIS Diagnostic Trade Integration Study

ESW Economic and Sector Work

MEF Ministry of Economy and Finance

PEMFAR Public Expenditure Management and Accountability Report

PER Public Expenditure Review

PRS Poverty Reduction Strategy 


\section{NIGER: TABLE OF COMMON INDICATORS REQUIRED FOR SURVEILLANCE}

(As of April 5, 2009)

\begin{tabular}{|c|c|c|c|c|c|c|c|}
\hline & \multirow{2}{*}{$\begin{array}{l}\text { Date of latest } \\
\text { observation }\end{array}$} & \multirow{2}{*}{$\begin{array}{l}\text { Date } \\
\text { received }\end{array}$} & \multirow{2}{*}{$\begin{array}{c}\text { Frequency } \\
\text { of } \\
\text { Data }^{6}\end{array}$} & \multirow{2}{*}{$\begin{array}{l}\text { Frequency } \\
\text { of } \\
\text { Reporting }\end{array}$} & \multirow{2}{*}{$\begin{array}{l}\text { Frequency } \\
\text { of } \\
\text { Publication }^{6}\end{array}$} & \multicolumn{2}{|c|}{ Memo Items: } \\
\hline & & & & & & $\begin{array}{c}\text { Data Quality - } \\
\text { Methodological } \\
\text { soundness } \\
\end{array}$ & $\begin{array}{l}\text { Data Quality - Accuracy } \\
\text { and reliability }^{8}\end{array}$ \\
\hline Exchange Rates & Current & Current & $\mathrm{D}$ & M & M & & \\
\hline $\begin{array}{l}\text { International Reserve Assets and Reserve Liabilities of } \\
\text { the Monetary Authorities }{ }^{1}\end{array}$ & Dec 2008 & Febr 2009 & M & 1 & M & & \\
\hline Reserve/Base Money & Dec 2008 & Febr 2009 & M & 1 & M & \multirow[t]{3}{*}{ LO, LO, LNO, LO } & \multirow[t]{3}{*}{$\mathrm{LO}, \mathrm{O}, \mathrm{O}, \mathrm{O}, \mathrm{NA}$} \\
\hline Central Bank Balance Sheet & Dec 2008 & Febr 2009 & M & 1 & M & & \\
\hline Consolidated Balance Sheet of the Banking System & Dec 2008 & Febr 2009 & M & 1 & M & & \\
\hline Interest Rates ${ }^{2}$ & Dec. 2008 & Febr 2009 & 1 & 1 & M & & \\
\hline Consumer Price Index & Febr. 2009 & Febr 2009 & M & M & M & O, LNO, O, O & LNO, O, O, LO, NA \\
\hline $\begin{array}{l}\text { Revenue, Expenditure, Balance and Composition of } \\
\text { Financing }^{3}-\text { General Government }\end{array}$ & Dec 2008 & March 2009 & & & & LO, LNO, O, LO & LO, O, O, O, NO \\
\hline $\begin{array}{l}\text { Stocks of Central Government and Central Government- } \\
\text { Guaranteed Debt }{ }^{5}\end{array}$ & Dec. 2008 & March 2009 & A & 1 & 1 & & \\
\hline External Current Account Balance & Dec. 2008 & March 2009 & A & I & A & \multirow[t]{2}{*}{$\mathrm{O}, \mathrm{O}, \mathrm{O}, \mathrm{LO}$} & \multirow[t]{2}{*}{ LO, LO, LO, O, LO } \\
\hline Exports and Imports of Goods and Services & Dec. 2008 & March 2009 & A & 1 & A & & \\
\hline GDP/GNP & Dec. 2008 & March 2009 & A & 1 & A & LO, LO, LNO, LO & LNO, LO, LNO, LO, NO \\
\hline Gross External Debt & Dec. 2008 & March 2009 & A & 1 & A & & \\
\hline
\end{tabular}

${ }^{1}$ Includes reserve assets pledged or otherwise encumbered as well as net derivative positions.

${ }^{2}$ Both market-based and officially determined, including discount rates, money market rates, rates on treasury bills, notes and bonds.

${ }^{3}$ Foreign, domestic bank, and domestic nonbank financing.

${ }^{4}$ The general government consists of the central government (budgetary funds, extra budgetary funds, and social security funds) and state and local governments.

${ }^{5}$ Including currency and maturity composition.

${ }_{7}^{6}$ Daily $(\mathrm{D})$; weekly (W); monthly (M); quarterly (Q); annually (A); irregular (I); and not available (NA).

Reflects the assessment provided in the data ROSC published on June 9, 2006, based on the findings of the mission that took place during March1-18, 2005. For the dataset corresponding to the variable in each row, the assessment indicates whether international standards concerning concepts and definitions, scope, classification/sectorization, and basis for recording are fully observed (O); largely observed (LO); largely not observed (LNO); not observed (NO); and not available (NA).

${ }^{8}$ Same as footnote 7, except referring to international standards concerning source data, assessment of source data, statistical techniques, assessment and validation of intermediate data and statistical outputs and revision studies. 
May 13, 2009

\section{IMF Completes the Second Reviews under Niger's PRGF and Approves US\$5.0 million Disbursement}

The Executive Board of the International Monetary Fund (IMF) completed today the second review of Niger's economic performance under the three-year Poverty Reduction and Growth Facility (PRGF) arrangement. The approval enables Niger to draw immediately an amount of SDR 3.29 million from the IMF (about \$5.0 million), bringing total disbursements to SDR 9.87 (about US\$15.0).

The PRGF arrangement became effective June 2, 2008, for a total amount of SDR 23.03 million (about US\$34.9 million) (see Press Release No. 08/127). The first review was completed on December 22, 2008. 